\title{
Inhibition of AAK1 Kinase as a Novel Therapeutic Approach to Treat Neuropathic Pain ${ }^{\mathbf{S}}$
}

\author{
Walter Kostich, ${ }^{1}$ Brian D. Hamman, ${ }^{1}$ Yu-Wen Li, Sreenivasulu Naidu, Kumaran Dandapani, \\ Jianlin Feng, Amy Easton, Clotilde Bourin, Kevin Baker, Jason Allen, Katerina Savelieva, \\ Justin V. Louis, Manoj Dokania, Saravanan Elavazhagan, Pradeep Vattikundala, \\ Vivek Sharma, Manish Lal Das, Ganesh Shankar, Anoop Kumar, Vinay K. Holenarsipur, \\ Michael Gulianello, Ted Molski, Jeffrey M. Brown, Martin Lewis, Yanling Huang, \\ Yifeng Lu, Rick Pieschl, Kevin O'Malley, Jonathan Lippy, Amr Nouraldeen, \\ Thomas H. Lanthorn, Guilan Ye, Alan Wilson, Anand Balakrishnan, Rex Denton,
} James E. Grace, Kimberley A. Lentz, Kenneth S. Santone, Yingzhi Bi, Alan Main, Jon Swaffield, Ken Carson, Sandhya Mandlekar, Reeba K. Vikramadithyan, Susheel J. Nara, Carolyn Dzierba, Joanne Bronson, John E. Macor, Robert Zaczek, Ryan Westphal, Laszlo Kiss, Linda Bristow, Charles M. Conway, Brian Zambrowicz, and Charles F. Albright

Research and Development, Neuroscience Biology (W.K., Y.-W.L., J.F., A.E., C.B., M.G., T.M., J.M.B., M.L., Y.H., Y.L., R.P., R.Z., R.W., L.K., L.B., C.M.C., C.F.A.), Neuroscience Chemistry (C.D., J.B., J.E.M.), Preclinical Candidate Optimization (A.B., J.E.G., K.A.L., K.S.S.), and Discovery Toxicology (R.D.), Bristol-Myers Squibb, Wallingford, Connecticut; Research and Development, Leads Discovery and Optimization, Bristol-Myers Squibb, Pennington, New Jersey (K.O., J.L.); BMS Biocon Research Center, Bangalore, India (K.D., J.V.L., S.N., M.D., S.E., P.V., V.S., M.L.D, G.S., A.K., V.K.H., S.M., R.K.V., S.J.N.); Lexicon Pharmaceuticals, The Woodlands, Texas (B.D.H., K.B., J.A., K.S., A.N., A.W., J.S., B.Z., T.H.L., G.Y.); and Lexicon Pharmaceuticals, Basking Ridge, New Jersey (Y.B., A.M., K.C.)

Received May 19, 2016; accepted July 6, 2016

\section{ABSTRACT}

To identify novel targets for neuropathic pain, 3097 mouse knockout lines were tested in acute and persistent pain behavior assays. One of the lines from this screen, which contained a null allele of the adapter protein- 2 associated kinase 1 (AAK1) gene, had a normal response in acute pain assays (hot plate, phase I formalin), but a markedly reduced response to persistent pain in phase II formalin. AAK1 knockout mice also failed to develop tactile allodynia following the Chung procedure of spinal nerve ligation (SNL). Based on these findings, potent, small-molecule inhibitors of AAK1 were identified. Studies in mice showed that one such inhibitor, LP-935509, caused a reduced pain response in phase II formalin and reversed fully established pain behavior following the SNL procedure. Further studies showed that the inhibitor also reduced evoked pain responses in the rat chronic constriction injury $(\mathrm{CCl})$ model and the rat streptozotocin model of diabetic peripheral neuropathy. Using a nonbrain-penetrant
AAK1 inhibitor and local administration of an AAK1 inhibitor, the relevant pool of AAK1 for antineuropathic action was found to be in the spinal cord. Consistent with these results, AAK1 inhibitors dose-dependently reduced the increased spontaneous neural activity in the spinal cord caused by $\mathrm{CCl}$ and blocked the development of windup induced by repeated electrical stimulation of the paw. The mechanism of AAK1 antinociception was further investigated with inhibitors of $\alpha 2$ adrenergic and opioid receptors. These studies showed that $\alpha 2$ adrenergic receptor inhibitors, but not opioid receptor inhibitors, not only prevented AAK1 inhibitor antineuropathic action in behavioral assays, but also blocked the AAK1 inhibitor-induced reduction in spinal neural activity in the rat $\mathrm{CCl}$ model. Hence, AAK1 inhibitors are a novel therapeutic approach to neuropathic pain with activity in animal models that is mechanistically linked (behaviorally and electrophysiologically) to $\alpha 2$ adrenergic signaling, a pathway known to be antinociceptive in humans.
This work was supported by Bristol-Myers Squibb and Lexicon Pharmaceuticals. All authors were employees of either Bristol-Myers Squibb or Lexicon Pharmaceuticals at the time when this work was done; many authors own in excess of $\$ 10,000$ of company stock. Additionally, some authors are inventors on patents related to the subject matter.

${ }^{1}$ W.K. and B.D.H. contributed equally to this work

dx.doi.org/10.1124/jpet.116.235333.

S This article has supplemental material available at jpet.aspetjournals.

\section{Introduction}

Neuropathic pain is caused by a lesion or disease of the somatosensory nervous system (reviewed in Costigan et al., 2009), such as herpes infection and diabetes, which can lead to postherpetic neuralgia and diabetic peripheral neuropathy, respectively. As a consequence of these conditions, patients can experience hyperalgesia (increased pain from a normally 
painful stimulus), allodynia (pain due to a stimulus that does not normally evoke pain), and spontaneous pain (pain arising without an obvious triggering event).

Neuropathic pain is commonly treated with tricyclic antidepressants, serotonin-norepinephrine reuptake inhibitors (SNRI), and gabapentinoids (Costigan et al., 2009; Finnerup et al., 2010). The antinociceptive mechanism of these medications is linked to the endogenous noradrenergic system, which is a powerful inhibitor of spinal dorsal horn circuits required for neuropathic pain (reviewed in Fairbanks et al., 2009). In particular, the endogenous system originates primarily from the locus ceruleus, where descending neurons project to the dorsal horn. When stimulated, these neurons release norepinephrine, which binds to $\alpha 2$ adrenergic receptors. Binding of norepinephrine to $\alpha 2 \mathrm{~A}$-adrenergic receptors on presynaptic afferent terminals reduces substance $P$ and glutamate release from primary afferents via the cholinergic pathways. Binding of norepinephrine to $\alpha 2 \mathrm{C}$-adrenergic receptors on postsynaptic secondary neurons causes hyperpolarization by $\mathrm{G}$ protein activation of G-protein gated inward rectifier potassium (GIRK) potassium channels. Gabapentinoids activate the descending inhibitory neurons in the locus ceruleus (Hayashida et al., 2008). In addition, gabapentinoids bind and affect the subcellular trafficking of $\alpha 2 \delta$-1 subunits of calcium channels, thereby preventing the pain-induced increase in calcium currents (Dolphin, 2012). SNRIs and tricyclic antidepressants cause antinociception by increasing the dorsal horn concentration of serotonin and norephinephrine by inhibiting their reuptake from the synapse (Benarroch, 2008). The $\alpha 2 \mathrm{~A}$ adrenergic and the $\alpha 2 \mathrm{C}$ adrenergic receptors can be activated directly with an $\alpha 2$ adrenergic agonist, such as clonidine. Although clonidine is used intrathecally as an opioid adjuvant for postoperative pain (Engelman and Marsala, 2013), broader clonidine usage for pain reduction is limited by sedation and hypotension. Consistent with a pathway linkage between these antinociceptives, $\alpha 2$ adrenergic antagonists prevent antinociception by gabapentin (Hayashida et al., 2007), SNRIs (Obata et al., 2005), and $\alpha 2$ adrenergic agonists (Fairbanks et al., 2009).

Despite the presence of marketed therapeutics for neuropathic pain, a significant unmet need remains. Importantly, the existing therapeutic compounds do not eliminate neuropathic pain for most patients (Finnerup et al., 2010). Specifically, less than half of neuropathic pain patients achieve a $50 \%$ reduction in pain with current treatments (Snedecor et al., 2014). Furthermore, current treatments frequently have undesired side effects such as weight gain from tricylic antidepressants and sedation plus cognitive impairment from gabapentinoids.

Given the significant unmet need, we screened knockout mice to identify novel pain targets. This strategy was based on a retrospective evaluation of the knockout phenotypes for the targets of the 100 best-selling drugs that showed that these phenotypes correlated well with known drug efficacy (Zambrowicz and Sands, 2003). The general phenotypic screen (Beltran del Rio et al., 2003; Tang et al., 2010), as well as the obesity (Brommage et al., 2008), diabetes (Powell et al., 2013), and bone (Brommage et al., 2014) components of this screen, was previously described, and datasets for 472 gene knockout lines were made publicly available (Tang et al., 2010). The pain component of this screen consisted of hot-plate and formalin-paw assays, both semiautomated assays amenable to screening large numbers of animals. The formalin assay assessed the inflammatory and central sensitization components in addition to the acute pain addressed by the hot-plate assay (Mogil, 2009). Using the formalin assay, AP2-associated kinase 1 (AAK1) knockouts were identified based on their reduced response to persistent pain. Based on these AAK1 knockout results, we created selective, small-molecule inhibitors of AAK1, a kinase implicated in endocytosis (Conner and Schmid, 2002). These AAK1 inhibitors recapitulated the knockout phenotype and reversed animal behaviors consistent with the relief of neuropathic pain. In addition, AAK1 inhibitors suppressed the increase in spontaneous action potentials following chronic constriction injury (CCI) nerve injury and prevented the development of windup normally induced by repeated electrical stimulation of afferents that innervate spinal dorsal horn cells. These AAK1 inhibitors were then used to behaviorally and electrophysiologically link the mechanism of pain reversal to adrenergic signaling in the spinal cord.

\section{Materials and Methods}

\section{Synthesis of AAK1 Inhibitors}

Experimental details and procedures for the synthesis of LP-935509, LP-922761, BMT-090605, BMT-124110, LP-927443, and BMS-901715 and full characterization (1H NMR, 13C NMR, low- or high-resolution mass spectrometry, optical rotation, and melting point) of final compounds are described in Supplemental Material.

\section{AAK1 Protein Expression and Biochemical Assays}

AAK1 protein was expressed using a recombinant baculovirus and purified, and kinase activity was determined using biochemical assays described in Supplemental Material. BMP-2-inducible protein kinase (BIKE) and Cyclin G-associated kinase (GAK) kinases were expressed, and kinase activity was determined, as described in Supplemental Material.

\section{AAK1 Cellular Assays}

AAK1 was transiently expressed in HEK293F cells together with adaptor protein 2 (AP-2), an AAK1 kinase substrate. The extent of inhibition of AP-2 phosphorylation at various compound concentrations was then determined by Western blotting. Detailed methods are described in Supplemental Material.

\section{AAK1 Knockout Behavior and In Vivo Pharmacology}

All of the animals were kept in an American Association for the Accreditation of Laboratory Animal Care-accredited animal holding facility maintained at controlled temperature $\left(23 \pm 1^{\circ} \mathrm{C}\right)$ and humidity $(50 \pm 20 \%)$ under a 12:12 hour light:dark cycle (lights on at 07:00 hours). Food and water were provided ad libitum. All experimental procedures were reviewed and approved by the Institutional Animal Ethics Committee and conducted in accordance with procedures set by the Committee for the Purpose of Control and Supervision on Experiments on Animals. AAK1 knockout mice were generated on a C57 $\times 129$ hybrid background strain. Exon 2 of the AAK1 gene was deleted using gene targeting by homologous recombination in 129/ SvEvBrd embryonic stem cells and confirmed by Southern blot. 
Chimeras were bred to C57BL/6J mice, and heterozygous mice were intercrossed. An additional null allele was produced by gene trapping (OST 23,964). Both alleles exhibited similar phenotypes (data not shown), and the work described in this paper was carried out with the gene-targeted allele. Mice used for LP-935509 in vivo pharmacology studies were of the C57BL/6J albino strain (Ye et al., 2015). The formalin-paw test was used to assess acute and tonic nociceptive responses in mice. A metal band was placed around the left hind paw of each mouse, and $20 \mu \mathrm{l} 5 \%$ formalin was s.c. injected in the dorsal surface of the left hind paw. Mice were individually housed in cylindrical chambers for 45 minutes. A computer recorded flinches per minute, total flinches for phase I (acute phase $=$ first 8 minutes), and total flinches for phase II (tonic phase between 20 and 40 minutes) through an electromagnetic field. In the primary screen, four knockout animals and four littermate controls for each line were tested to identify potential phenotypes that could then be followed up in larger cohorts. Some knockout lines were not screened due to embryonic lethality or timing of implementation of the formalin test.

\section{LP-935509 Drug Administration}

For all mouse studies, LP-935509 was formulated in 10\% Cremophor and dosed orally at 10,30 , and $60 \mathrm{mg} / \mathrm{kg}(10 \mathrm{ml} / \mathrm{kg})$. Gabapentin (G154Sigma from Sigma-Aldrich, St. Louis, MO) was freshly dissolved in sterile saline and administered orally at $200 \mathrm{mg} / \mathrm{kg}(10 \mathrm{ml} / \mathrm{kg})$. Both drugs were given 30 minutes prior to the formalin injection or prior to von Frey testing. Mice were tested for baseline von Frey sensitivity 24 hours prior to the validation study. The mice that met the following selection criteria were included in the drug validation experiment: 1) von Frey 50\% threshold for ipsilateral paw $<1$, and 2) $50 \%$ threshold for contralateral paw $>2$. Von Frey testing was performed at 0,30 , and 120 minutes after LP-935509 dosing.

For all rat studies [with the exception of the pharmacokinetic (PK) and rotarod experiments], LP-935509 was formulated in $40 \%$ polyethylene glycol, $10 \%$ ethanol, $15 \%$ Tween 80 , and $35 \%$ water and delivered orally at the specified dose and a volume of $3 \mathrm{ml} / \mathrm{kg}$. In the PK and rotarod studies, LP-935509 was prepared in 10\% Cremophor EL in water. Gabapentin was formulated in $40 \%$ polyethylene glycol, $10 \%$ ethanol, $15 \%$ Tween 80 , and $35 \%$ water for all rat studies, except the rotarod study, in which case it was prepared in 25\% Captisol, $0.1 \mathrm{M}$ citrate buffer ( $\mathrm{pH} 3.0)$.

\section{Administration of Other Drugs}

Morphine and naloxone were dissolved in saline and each delivered s.c. at $3 \mathrm{mg} / \mathrm{kg}$ and $1 \mathrm{mg} / \mathrm{kg}$, respectively, in a volume of $3 \mathrm{ml} / \mathrm{kg}$. Yohimbine and tizanidine were dissolved in saline and each delivered i.p. at $1 \mathrm{mg} / \mathrm{kg}$ in a volume of $2 \mathrm{ml} / \mathrm{kg}$. BMT-090605 and clonidine were dissolved in artificial cerebrospinal fluid with HEPES buffer in preparation for intrathecal dosing. Final dosing volume for both was $10 \mu$ l. LP-922761 was formulated in 10\% Cremophor and dosed orally for the mouse spinal nerve ligation (SNL) study (10 $\mathrm{ml} / \mathrm{kg})$.

\section{Open-Field Assay in Mice}

Mice were acclimated to the experimental room illuminated at 30 lux for 30 minutes. Mice were then placed into the testing chamber containing infrared sensors along the perimeter (Montana et al., 2011). Locomotor activity, counted with each beam break, was recorded for 30 minutes using automated software (Accuscan). The sum activity detected over the 30-minute period was used for statistical analysis. The chambers were cleaned with $70 \%$ ethanol between test sessions.

\section{Formalin Assay in Mice}

Persistent pain or hyperalgesia can be induced with an injection of formalin into the dorsum of the hind paw, as previously described (Dubuisson and Dennis, 1977). To measure hyperalgesia in AAK1 knockout mice, a metal band was placed around the right hind paw of each mouse prior to testing. After a 30-minute acclimation period to an individual cylindrical test chamber, $20 \mu \mathrm{l} 5 \%$ formalin in saline (Sigma-Aldrich) was s.c. injected in the dorsal surface of the right hind paw. Mice were immediately placed back into the test chamber where movement of the metal band breaks the electromagnetic field of a loop antenna located under the mouse and is automatically recorded as a flinch. The receiver output is amplified, filtered, and digitized for analysis using the Automated Nociception Analyzer (Yaksh et al., 2001) (Ozaki laboratory, University of California, San Diego, CA). Flinches were recorded for 60 minutes. The mouse displays a biphasic incidence of flinching (phase I or acute phase was defined as 0-9 minutes, and phase II or tonic phase was defined as 10-60 minutes, as indicated), and the data from each phase are summed for statistical analysis and graphical representation. For testing compounds, slight modifications were made to the protocol. In these studies, investigatory compounds were administered 30 minutes prior to formalin injection. The left hind paw was injected with fresh $5 \%$ formalin prepared by diluting formaldehyde (Formalde-fresh $20 \%$; Fisher Scientific, Fair Lawn, NJ) with distilled water. Phase II data included the sum of flinches between 20 and 40 minutes after the formalin injection.

\section{SNL in Mice}

Methods for mouse SNL were carried out according to the procedures devised by Kim and Chung (1992) with modifications, as published previously (Kim and Chung, 1992; Ye et al., 2015). At 5-7 weeks of age, male mice were anesthetized with isoflurane ( $2 \%$ at the oxygen flow rate of $1 \mathrm{ml} / \mathrm{min})$. A skin incision $(1 \mathrm{~cm})$ was made $1 \mathrm{~mm}$ to the left of the dorsal midline, using the level of iliac crests as the midpoint of the incision. The paraspinal muscles were bluntly separated medial to the iliac crest to reveal transverse processes between the caudal edge of lumbar level four (L4) and the rostral edge of L6 [or sacroiliac junction in mice that had only five lumbar vertebrae $(\mathrm{LV})$ ]. This approach facilitated identification of spinal nerves L4, L5, and/or L6. The position of the last two lumbar transverse processes in relation to the iliac crest was used to differentiate mice with five LV from those with six. Using these bone landmarks, the number of LV that a mouse possesses was accurately identified in the majority of cases. Ligation of the L4 and L5 spinal nerves was performed by passing a suture under L5 with one pair of fine forceps and pulling the suture out from the other side with another pair of fine forceps. After hemostasis was confirmed, the incision was closed in two layers, with 5-0 vicryl suture for the dorsolumbar fascia and wound clips for the skin. In sham-operated animals, the surgical procedure was identical to that as described above, except that spinal nerves were not ligated. Mice were given an injection of saline $(1 \mathrm{ml})$ and buprenorphine $(0.05-0.1 \mathrm{mg} / \mathrm{kg}$ mice $)$ immediately following the surgery and buprenorphine again at approximately 12 and 24 hours postsurgery (for a total of three doses) to relieve surgery-induced pain. A warming pad at $36 \pm 1{ }^{\circ} \mathrm{C}$ and a heating lamp were used to maintain normal body temperature of the animal throughout the surgery. Mice were individually housed after the procedure and were monitored until complete recovery from anesthesia. For drug studies, mice were tested 3-4 weeks postsurgery.

\section{Mechanical Allodynia in Mice via Manual von Frey}

Mechanical allodynia was assessed by testing the hind paw withdrawal response (withdrawal, flinching, licking) to a set of von Frey filaments (numbered 2.44, 2.83, 3.22, 3.61, 4.08, and 4.31 corresponding approximately to force of $0.04,0.07,0.16,0.4,1$, and $2 \mathrm{~g}$; Stoelting, Wood Dale, IL) in an up-down procedure, as described by Chaplan et al. (1994). Baseline von Frey tests were carried out 24 hours prior to surgery and repeated once per week for 3-6 weeks after surgery depending on experimental design. For the von Frey tests, mice were placed in transparent polyethylene terephthalate cylinders with a wire mesh floor to allow the experimenter to apply the 
von Frey filament to the mouse plantar surface. Both paws were tested. The first von Frey filament applied was 3.61. If no response was elicited, the next stronger filament was presented. If there was a response, the next weaker filament was presented. There were total of six presentations of von Frey filaments. If the animal did not respond to the strongest filament, 4.31, the test was ended. The 50\% withdrawal threshold was calculated for each paw using the up-down method. Mice that exhibited 50\% withdrawal threshold below 2 in any paw during baseline testing were excluded from surgery and further assessment. Von Frey test experimenters were always blind to the nature of the surgery and the treatment.

\section{$\mathrm{CCl}$ in Rats}

On the day of surgery, male Sprague-Dawley rats were transferred from the holding area to an aseptic surgery suite. Animals were anesthetized with $2 \%$ isoflurane and placed in the prone position, and the left hind limb was supported and immobilized with adhesive tape. Following shaving of the fur and disinfection of the surgical site with betadine, a small incision was made in the midposterior thigh to expose the sciatic nerve. Four ligations, made of 4-0 chromic gut, were loosely tied around the nerve 1-2 mm apart without interrupting the epineural blood supply (Bennett and Xie, 1988). A sham group of rats underwent a similar surgical procedure but without the nerve ligation. On completion of the procedure, the incision was closed with surgical thread, topical disinfectant (povidone-iodine solution i.p.) was applied locally, and Gentamicin (4 mg/kg) was administered s.c. Animals were initially housed individually and then pair-housed 3 days after surgery until behavioral testing. Separate groups of rats were evaluated for thermal hyperalgesia, cold allodynia, mechanical hyperalgesia, or mechanical allodynia 2-4 weeks postsurgery.

\section{Streptozotocin-Induced Diabetic Peripheral Neuropathic Pain in Rats}

Male Sprague-Dawley rats were fasted overnight and then treated with $50 \mathrm{mg} / \mathrm{kg}$ streptozotocin (STZ) prepared in $0.1 \mathrm{M}$ citrate buffer (pH 4.5) (Courteix et al., 1993). Rats were allowed food 1 hour after STZ treatment and were monitored for the next 48 hours for any symptoms of hypoglycemia. Animals were evaluated for mechanical allodynia beginning 5-6 weeks post-STZ administration. Hyperglycemia, defined as a plasma glucose level $>250 \mathrm{mg} / \mathrm{dL}$, was confirmed in all subjects prior to testing.

\section{Thermal Hyperalgesia in Rats}

Thermal hyperalgesia was measured using the Hargreaves plantar test (Hargreaves et al., 1988). On the test day, CCI rats were acclimatized for 1 hour to the experimental room and then placed in observational boxes situated on a temperature-regulated platform maintained at $30^{\circ} \mathrm{C}$ for 30 minutes. The paw withdrawal latency following application of a radiant heat source of $35 \%$ intensity was measured for three trials (intertrial interval $=5-10$ minutes), and the average latency was calculated at each time point for each subject. To prevent tissue damage, a cutoff time of 30 -second radiant heat application was used.

\section{Cold Allodynia in Rats}

Cold allodynia was assessed using a cold- and hot-plate antinociception meter (IITC Life Science Incremental Hot Cold Plate Analgesia Meter). On the test day, rats were acclimatized for 1 hour to the experimental room and then placed into an observational box for 5 minutes. The latency to the first brisk lift/withdrawal or licking/ guarding of the hind paw was recorded at $10^{\circ} \mathrm{C}$. A general limb movement involving coordinated movement of all four limbs (e.g., walking) was not considered as a withdrawal response. To prevent tissue damage, a maximum cutoff time limit of 100 seconds was used (Tanimoto-Mori et al., 2008).

\section{Mechanical Allodynia in Rats}

Manual von Frey. For CCI studies, rats were acclimatized for 1 hour to the experimental room and then placed into observational boxes with wire-mesh floors for 20 minutes. Paw withdrawal threshold was recorded using a series of von Frey hairs with a force of $0.4 \mathrm{~g}, 0.8 \mathrm{~g}$, $1.5 \mathrm{~g}, 2.5 \mathrm{~g}, 4 \mathrm{~g}, 8 \mathrm{~g}, 10 \mathrm{~g}$, and $20 \mathrm{~g}$ (IITC Life Science, Woodland Hills, CA; model 2390 series) applied perpendicularly to the midplantar surface (Chaplan et al., 1994). Brisk paw withdrawal or licking immediate after pressure application was defined as a positive response, and lack of paw withdrawal within 6 seconds was defined as a negative response. Ambulation was considered an ambiguous response, and in such cases the stimulus was repeated. Both CCI/ sham groups of rats were evaluated for paw withdrawal threshold 2 weeks postsurgery.

Electronic von Frey. For STZ studies, rats were first acclimatized for 1 hour to the experimental room and then placed into observational boxes with wire-mesh floors for 20 minutes. Paw withdrawal threshold was recorded using an electronic von Frey Aesthesiometer (model no. series 2390; IITC Life Science). The rigid electronic von Frey hair was applied to the midplantar surface with an ascending force for 6 seconds. The maximum force at which the animal shows a withdrawal/licking response was recorded as the paw withdrawal threshold, as shown on the digital display. Withdrawal thresholds for each hind paw were measured in two trials with an intertrial interval of 5 minutes. The mean paw withdrawal threshold (g) of all four trials was calculated for each time point (Morrow, 2004).

\section{Mechanical Hyperalgesia in Rats}

Rats were handled before commencing the study, placed in a sling suit, and allowed to acclimatize for 20 seconds. Mechanical hyperalgesia was assessed using a digital paw pressure Randall Selitto instrument (IITC Life Science). An incremental pressure was applied to the plantar surface of the paw. Increasing force was applied until a vocalization/withdrawal response was observed with a $250 \mathrm{~g}$ cutoff applied to prevent tissue damage. The withdrawal threshold was measured in two trials (5-minute intertrial interval), and all four readings were averaged to determine the mean nociceptive threshold (g) (Santos-Nogueira et al., 2012).

\section{Rat Tail-Flick Assay}

Rats were transferred to the experimental room to acclimate for 1 hour and then selected randomly for testing (D'Amour and Smith, 1941). A radiant heat source (Panlab, LE7106 analgesia meter) was focused on the marked area of tail approximately $5 \mathrm{~cm}$ from the distal end of the tail while the animals were held. When the animal flicked his tail in response to the heat, the instrument stopped radiation and the time was noted as the tail-flick latency. Three latency measurements were taken per animal separated by at least 5 minutes and were averaged to determine the final latency. A maximum cutoff time of 10 seconds was used to avoid injury. Animals were tested 30 minutes after morphine administration and 90 minutes after LP-533509 or vehicle administration. To evaluate the analgesic response, pretreatment latency was compared with post-treatment latency using paired $t$ test. Data are expressed as the mean \pm S.E.M. with $P<0.05$ being considered statistically significant.

\section{Rat Hot-Plate Assay}

Animals were acclimatized to the hot plate for 15 minutes 1 day before the test (Woolfe and MacDonald, 1944). On the test day, individual rats were placed on a hot plate (BIOSEB) $55 \pm 1^{\circ} \mathrm{C}$ with a cutoff time of 30 seconds. Latency to response, such as lifting or licking a hind paw, jumping, or vocalization, was recorded. Baseline latency was recorded before the treatment. Animals were then administered morphine, LP-935509, or vehicle, and the latency was recorded 30 minutes (morphine) or 90 minutes (LP-935509 and vehicle) postdosing. Three latencies were measured at a minimum of 
5-minute intervals and were averaged to determine the final latency. To evaluate the analgesic response, pretreatment latency was compared with post-treatment latency using paired $t$ test. Data are expressed as the mean \pm S.E.M. with $P<0.05$ being considered statistically significant.

\section{Rotarod Assay}

Rotarod performance was measured using a Rotamex 5 instrument in male naive Sprague-Dawley rats (230-250 g) (Dunham and Miya, 1957; Watzman et al., 1964). Rats were trained for 3 consecutive days on the accelerating rod for 5 minutes. The training session consisted of two trials per day, one each in the morning and in the afternoon, in which the rat was placed on a horizontally oriented accelerated rotating rod configured to accelerate at a speed of 2-20 rpm over 5 minutes. Each day's second training session ended with an additional training of 30 seconds at $0-14 \mathrm{rpm}$ speed. On the test day, the rats were acclimated to the testing room for 1 hour, followed by dosing with vehicle or drug. At 1,3 , and 5 hours post-treatment, rats were placed on the accelerating rotarod, and time spent on the rotating beam before falling was recorded in a 5-minute test session. Animals able to remain on the rotarod for the entire test session were given a score of 300 seconds.

\section{Intrathecal Injection}

A rat was held firmly from the pelvic girdle with one hand while a $25 \mu \mathrm{l}$ glass Hamilton syringe with 25-ga needle was positioned and inserted between L4 and L5 of the spinal process at $75-80^{\circ}$ angle relative to taut skin plane (Hylden and Wilcox, 1980; Mestre et al., 1994). Once the injector sensed the bone, the needle was lowered to an approximately $30^{\circ}$ angle and slipped between the vertebrae (Fairbanks, 2003). The solution was then injected very slowly in a volume of $10 \mu \mathrm{l}$, and the needle rotated on withdrawal. The tip of the needle was inserted in such a way as approximately $0.5 \mathrm{~cm}$ was within the vertebral column. Test compounds were prepared in artificial cerebrospinal fluid with HEPES buffer.

\section{In Vivo Spinal Electrophysiology}

Rats were anesthetized with isoflurane (5\% induction, 2-3\% maintenance) and paralyzed with pancuronium ( $1 \mathrm{mg} / \mathrm{kg}$ i.v.). Animals were artificially ventilated, and the arterial pressure was monitored. A laminectomy was performed at the L1-5 vertebral level to expose the spinal cord, and a carbon fiber-recording electrode (Kation Scientific, Minneapolis, MN)) was inserted into lamina V of the dorsal horn using a SCAT microdrive (FHC, Bowdoin, ME). Extracellular action potential spikes (either spontaneous or evoked) from single neurons were isolated, amplified, and filtered using ExAmp-20KB (Kation Scientific). The spike activities were acquired and analyzed using a CED 1401 interface and Spike 2 software (Cambridge Electronic Design, Cambridge, UK). In experiments in which CCI rats were used, electrophysiological recordings were carried out in the rats $2-5$ weeks postsurgery. In these animals, spontaneous action potential spikes were recorded in the dorsal horn ipsilateral to the side of the sciatic ligation, and spike frequencies were determined. In the windup studies in which naive rats were used, wide-dynamic range cells in the dorsal horn were identified according to their responses to innocuous mechanical (brushing hind paw skin) and noxious (pinching of the skin) stimuli. Trains of electrical stimuli (16 pulses, 2.0 -ms duration, $2 \mathrm{nA}$ intensity, 5-minute interval) were applied through a pair of fine needle electrodes inserted s.c. into the receptive field of the plantar skin, and the stimulus intensity was set to activate $\mathrm{C}$-fiber-mediated spike activation.

LP-935509 and yohimbine were administrated by i.v. bolus injections. For the microiontophoresis test, double-barrel microelectrodes were inserted to the dorsal horn with one (carbon electrode) to record single neuronal spike activity and another (glass barrel) to focally administer BMT-124110 by microiontophoresis (30 mM, pH 4, +10-30 $\mathrm{nA}, 3$ minutes).

\section{Results}

AAK1 Knockout Mice Have an Antinociceptive Phenotype in the Formalin Assay and SNL Model. To identify novel therapeutic targets for neuropathic pain, 3097 homozygous mouse knockout lines representing the druggable genome (Brommage et al., 2014) were tested in the formalin assay (Dubuisson and Dennis, 1977). In this assay, mice are injected in one hind paw with dilute formalin, and the behavioral response of hind paw flinches is observed. Quantifying the number of flinches over time thereby provides a measure of pain behavior. Flinches are further divided into those occurring within the first 10 minutes (phase I), which primarily represent an acute pain state, and those that occur
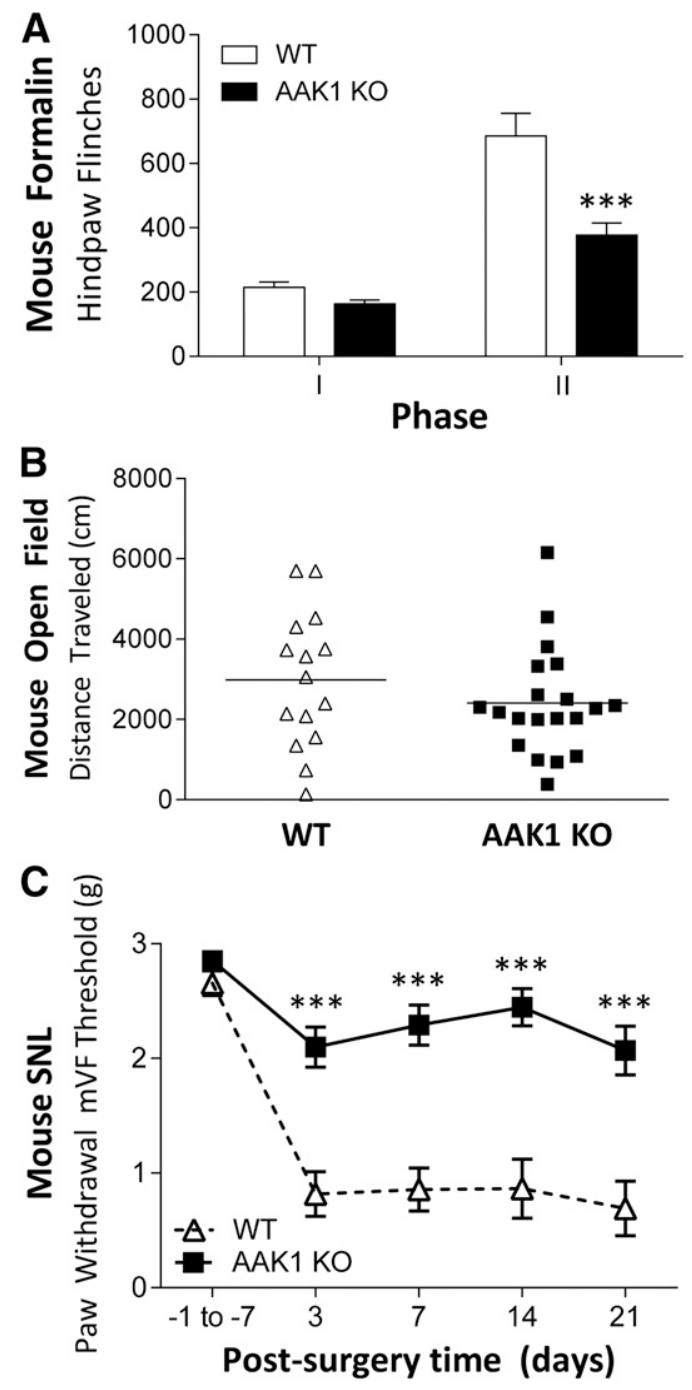

Fig. 1. AAK1 knockout mice have an antinociceptive phenotype in the formalin assay and SNL model. (A) Hind paw flinches in formalin phase I and phase II from wild-type (WT) and AAK1 knockout (AAK1 KO) mice $(n=$ 25-56 per group, male and female mice distributed equally between groups). *** $P<0.001$ versus WT by two-way repeated measures analysis of variance, followed by Bonferroni's post hoc test. (B) Distance traveled in open field testing of WT $(n=15)$ and AAK1 KO mice $(n=20)$. Male and female mice were used and distributed equally between groups. $P=0.27$ unpaired $t$ test. (C) Mechanical allodynia measured using manual von Frey $(\mathrm{mVF})$ fibers after SNL surgery for WT $(n=15)$ and AAK1 KO mice $(n=22)$. Male and female mice were used and distributed equally between groups. $* * * P<0.001$ versus WT by two-way repeated measures analysis of variance, followed by Bonferroni's post hoc test. 
between 10 and 60-90 minutes (phase II), which reflect a tonic pain state similar to the relatively long-lasting stimuli in human disease (Dubuisson and Dennis, 1977), such as neuropathic pain. The AAK1 knockout line exhibited a reduced number of flinches in the phase II formalin response (Fig. 1A). This reduced formalin response was not due to suppressed motor function (which can masquerade as lessened pain behavior) because AAK1 knockout mice were indistinguishable from wild-type animals in the open-field assay (Fig. 1B).

The AAK1 knockout mice and wild-type control mice were then tested in a SNL model commonly used to evaluate treatments for neuropathic pain (Kim and Chung, 1992; Ye et al., 2015). In this model, ligation of the L4 and L5 spinal nerves resulted in mechanical allodynia, a painful response to a normally benign tactile stimulus, which developed by 3 days postsurgery and persisted for at least 3 weeks in wild-type mice (Fig. 1C). In contrast to wild-type mice, AAK1 knockout mice did not develop mechanical allodynia following surgery over this same postsurgery period (Fig. 1C). These data were consistent with the lack of persistent pain response in the formalin assay and indicate that AAK1 plays a role in the development of persistent and neuropathic pain states.

A broad phenotypic screen was used to look for other phenotypes in the AAK1 knockout mice (Beltran del Rio et al., 2003). No consistent phenotypes were observed beyond the pain phenotypes described.

A review of the scientific literature indicated that the AAK1 protein was a serine/threonine protein kinase that was implicated in endocytosis (Conner and Schmid, 2002). Endocytosis via clathrin-coated vesicles is a major mechanism to move cell surface proteins to endosomes or lysosomes for recycling or degradation, respectively (reviewed in Traub, 2009). Cell surface proteins are targeted for clathrin-mediated endocytosis by binding the AP-2 adaptor complex. AP-2 consists of four proteins, including the $\alpha$ subunit, $\beta 2$ subunit, $\mu 2$ subunit, and $\sigma 2$ subunit. A variety of sorting signals is used for recognition by the AP-2 complex; one such recognition signal is binding of the $\mu 2$ subunit to the YXXPhi motif in proteins, where $\mathrm{X}$ is any amino acid and Phi is a bulky hydrophobic amino acid. The AAK1 kinase phosphorylates threonine 156 of the $\mu 2$ subunit, inducing a conformational change that facilitates binding of YXXPhi motifs and thereby enhances endocytosis (Jackson et al., 2003).

Identification of an AAK1 Inhibitor. The absence of a pain phenotype for AAK1 knockout mice in the SNL model led us to hypothesize that an AAK1 kinase inhibitor might be an effective way to reduce neuropathic pain. To test this hypothesis, selective, small-molecule inhibitors of AAK1 suitable for in vivo studies were identified. In particular, a highthroughput screen and subsequent lead optimization effort led to the identification of a potent and orally available AAK1 inhibitor LP-935509 (Fig. 2A). In an enzyme assay using the human AAK1 kinase domain (amino acids 30-330), LP-935509 inhibited phosphorylation of a peptide derived from the $\mu 2$ protein with an $\mathrm{IC}_{50}$ value of $3.3 \pm 0.7 \mathrm{nM}(n=8)$ (Supplemental Fig. 1A). To test the activity of LP-935509 in an intact cellular system, the compound was incubated with HEK293 cells overexpressing human AAK1 and the human $\mu 2$ protein. These studies showed that LP-935509 inhibited $\mu 2$ phosphorylation with an $\mathrm{IC}_{50}$ value of $2.8 \pm 0.4 \mathrm{nM}(n=26)$ (Supplemental Fig. 1B; Table 1). Additional enzymatic studies showed that LP-935509 was competitive with ATP with a Ki of $0.9 \mathrm{nM}$ (Supplemental Fig. 2).

To better understand LP-935509 kinase selectivity, we evaluated the effect of LP-935509 on the kinase activity of the two most closely related kinases, BIKE and GAK ( $77 \%$ and $39 \%$ amino acid identity versus AAK1 in the kinase domain,

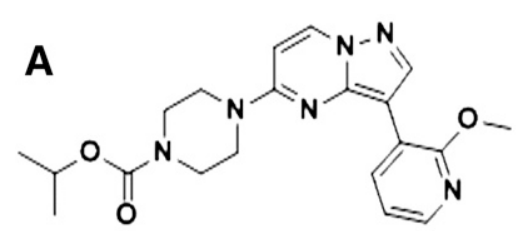

LP-935509
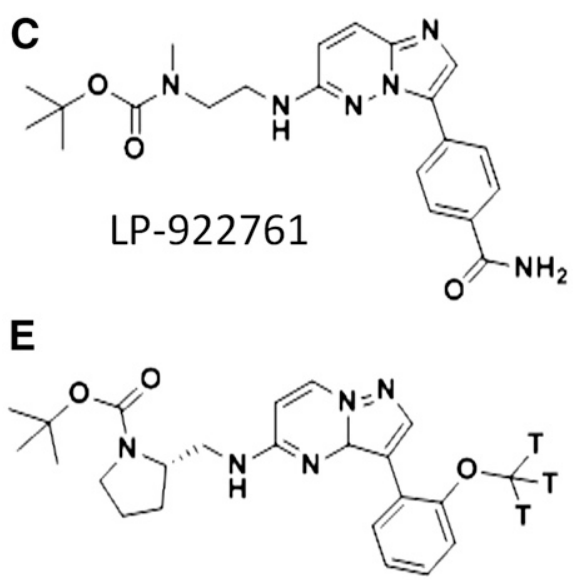

LP-927443
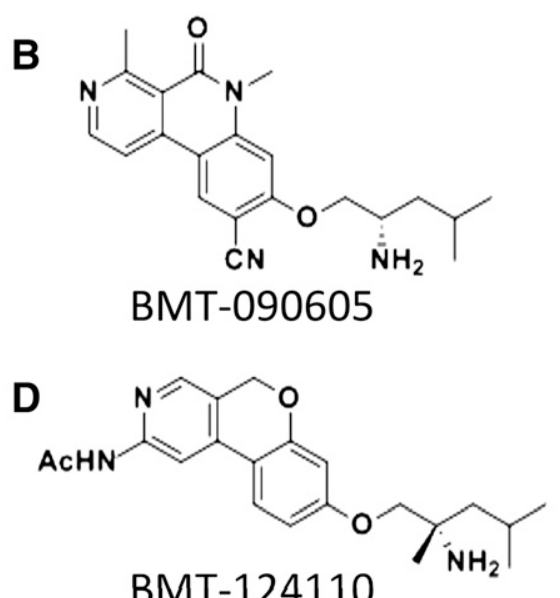

Fig. 2. Chemical structure of AAK1 inhibitors. (A) LP-935509. (B) BMT-090605. (C) LP-922761.

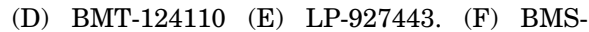
901715<smiles></smiles> 
TABLE 1

Summary of in vitro properties of AAK1 inhibitors

\begin{tabular}{lccrr}
\hline \multirow{2}{*}{ Assay } & LP-935509 & LP-922761 & BMT-090605 & BMT-124110 \\
\cline { 2 - 5 } & \multicolumn{4}{c}{ Average IC $_{50} \pm$ S.E.M. $(n), \mathrm{nM}$} \\
\hline AAK1 enzyme & $3.3 \pm 0.7(8)$ & $4.8(2)$ & $0.6 \pm 0.1(9)$ & $0.9 \pm 0.2(7)$ \\
AAK1 binding & $3.3 \pm 1.5(4)$ & $3.6(2)$ & $0.4 \pm 0.1(4)$ & $1.0(1)$ \\
AAK1 cell assay & $2.8 \pm 0.4(26)$ & $7.6 \pm 0.7(58)$ & $0.6 \pm 0.4(4)$ & $3.2 \pm 1.4(2)$ \\
BIKE enzyme & $14(1)$ & $24(1)$ & $45(1)$ & $17(1)$ \\
GAK enzyme & $320 \pm 40(2)$ & $\mathrm{NA}$ & $60(1)$ & $99(1)$ \\
$\mu$ Opioid receptor binding & $>30,000(1)$ & $15,000(1)$ & $>30,000(1)$ & $>30,000(1)$ \\
$\kappa$ Opioid receptor binding & $>30,000(1)$ & $>30,000(1)$ & $29,000(1)$ & $>30,000(1)$ \\
Adrenergic $\alpha 2$ 2A receptor binding & $>30,000(1)$ & $>30,000(1)$ & $>30,000(1)$ & $>30,000(1)$ \\
Adrenergic $\alpha 2 C$ receptor binding & $>30,000(1)$ & $>30,000(1)$ & $>30,000(1)$ & $15,000(1)$ \\
Adrenergic $\alpha 2$ 2A functional & $>1,000(4)$ & NA & $>10,000(4)$ & NA \\
Adrenergic $\alpha 2$ 2C functional & $>1,000(3)$ & NA & $>10,000(3)$ & NA \\
GABAa $\alpha 1$ receptor modulator & $12,000(3)$ & $>30,000(1)$ & $>30,000(1)$ & $>30,000(1)$ \\
GABAa $\alpha 2$ receptor modulator & $12,000(3)$ & NA & NA & NA \\
\hline
\end{tabular}

respectively). These studies showed that LP-935509 was a potent inhibitor of $\mathrm{BIKE}\left(\mathrm{IC}_{50}=14 \mathrm{nM}\right)$ and a modest inhibitor of $\mathrm{GAK}\left(\mathrm{IC}_{50}=320 \pm 40 \mathrm{nM}\right)$. To test additional kinases, the ability of $1 \mu \mathrm{M}$ LP-935509 to inhibit the binding of 389 kinases to an ATP-binding probe was measured. These studies showed that LP-935509 inhibited more than $70 \%$ of probe binding to 13 kinases, including BIKE (Supplemental Table 1). None of these other LP-935509-binding kinases have a known connection to pain modulation. In addition, the comparison of kinase profiling data across several of our other AAK1 inhibitors indicated a lack of consistent inhibition at any other kinase aside from BIKE (data not shown).

To further understand the pharmacology of LP-935509, the compound was tested at multiple concentrations in functional or binding assays for 43 receptors, transporters, and enzymes (Supplemental Table 2). LP-935509 was inactive at up to $10 \mu \mathrm{M}$ in the vast majority of the assays (42 of 43), with the most potent interaction observed for phosphodiesterase 4 (PDE4) $\left(\mathrm{IC}_{50}=8.4 \mu \mathrm{M}\right)$. Several receptors and transporters associated with nociception were among those evaluated, including the following: $\kappa$ and $\mu$ opioid receptors (binding $\left.\mathrm{IC}_{50}>30 \mu \mathrm{M}\right), \operatorname{NET}\left(\mathrm{IC}_{50}=22 \mu \mathrm{M}\right), \operatorname{SERT}\left(\mathrm{IC}_{50}>30 \mu \mathrm{M}\right)$, $\alpha 2 \mathrm{~A}$ and $\alpha 2 \mathrm{C}$ adrenergic receptors (binding $\mathrm{IC}_{50}>30 \mu \mathrm{M}$ ), and positive modulation of $\gamma$-aminobutyric acid (GABA)a $\alpha 1-$ and GABAa $\alpha 2$-containing channels $\left(\mathrm{EC}_{50}=12 \mu \mathrm{M}\right.$ and $12 \mu \mathrm{M}$, respectively).

Taken together, these studies show that LP-935509 is a potent and selective AAK1 kinase inhibitor. Although the inhibition of BIKE could contribute to nociception, it is unlikely that other pharmacologic activities of LP-935509 are relevant for nociception.

An AAK1 Inhibitor Recapitulates the AAK1 Knockout Antinociceptive Phenotype in Mice. To facilitate the use of LP-935509 in mouse behavioral studies, the pharmacokinetic properties of LP-935509 in mice were quantified. For these studies, four mice were dosed with LP-935509 either i.v. $(1 \mathrm{mg} / \mathrm{kg}$ ) or orally $(10 \mathrm{mg} / \mathrm{kg})$. Blood samples were taken over a 24-hour period, and LP-935509 plasma levels were determined (Supplemental Fig. 4). PK parameters were fit to standard PK models. This analysis showed that LP-935509 had $100 \%$ oral bioavailability and a plasma half life of 3.6 hours. The Cmax for the $10 \mathrm{mg} / \mathrm{kg}$ oral dose was $5.2 \mu \mathrm{M}$ at 0.5 hour postdose. In addition, LP-935509 had a plasma-free fraction of $2.6 \%$ in mice. Brain drug levels exceeded plasma drug levels with a brain/plasma drug ratio typically between
3 and 4, showing that LP-935509 was highly brain-penetrant. Based on these data, LP-935509 was considered a suitable compound for oral delivery to evaluate the effect of AAK1 inhibition on nociception.

The effect of LP-935509 on the formalin phase II responses was tested to determine whether a small-molecule AAK1 inhibitor could recapitulate the AAK1 knockout phenotype. These studies showed that oral delivery of LP-935509 caused a dose-dependent reduction in phase II paw flinches that was significantly lower than the vehicle-treated animals (Fig. 3A). In particular, $30 \mathrm{mg} / \mathrm{kg}$ and $60 \mathrm{mg} / \mathrm{kg}$ LP-935509 caused a robust reduction in pain behavior similar to a high dose of gabapentin (200 mg/kg) (Fig. 3A). LP-935509 was then tested in the mouse SNL model, and oral delivery exhibited a dosedependent reversal of the mechanical allodynia, with $60 \mathrm{mg} / \mathrm{kg}$-treated mice displaying behavioral responses to tactile stimuli similar to a presurgery mouse by 120 minutes postdose (Fig. 3B). As expected, gabapentin $(200 \mathrm{mg} / \mathrm{kg})$ also reduced mechanical allodynia in this assay. Unlike high doses of gabapentin, which are known to cause motor impairment and sedation, the effects of LP-935509 in the mouse formalin and SNL assays were not due to decreased locomotor activity as mice treated orally with the same doses of LP-935509 were indistinguishable from vehicle-treated animals in the openfield assay (Fig. 3C).

LP-935509 plasma and brain drug levels were measured in satellite animals during the mouse formalin, SNL, and open field tests (Supplemental Table 3). These measurements showed that brain drug levels exceeded plasma drug levels, showing that LP-935509 is highly brain-penetrant and suggesting that LP-935509 broadly distributed to tissues. For the mouse formalin and SNL assays (Fig. 3, A and B), LP-935509 reversed approximately half of the behavioral deficit at $10 \mathrm{mg} / \mathrm{kg}$, which corresponded to free drug levels in satellite animals at 30-120 minutes that were about 30-fold higher than the cellular $\mathrm{IC}_{50}$ value of $2.7 \mathrm{nM}$ (Supplemental Table 3 ).

To better understand the relationship of LP-935509 efficacy to exposure, the compound was dosed in the SNL mice at 3, 10, and $30 \mathrm{mg} / \mathrm{kg}$, and testing was carried out at several times, concluding with a final time of 24 hours (Fig. 4). LP-935509 plasma exposures were determined using satellite mice. Efficacy peaked by 2 hours, declined by 5 hours, and was absent by 24 hours. Total plasma exposures were highest at the 30 minutes and declined by 24 hours to less than $2 \%$ of the peak exposure. These data suggest that efficacy is correlated 


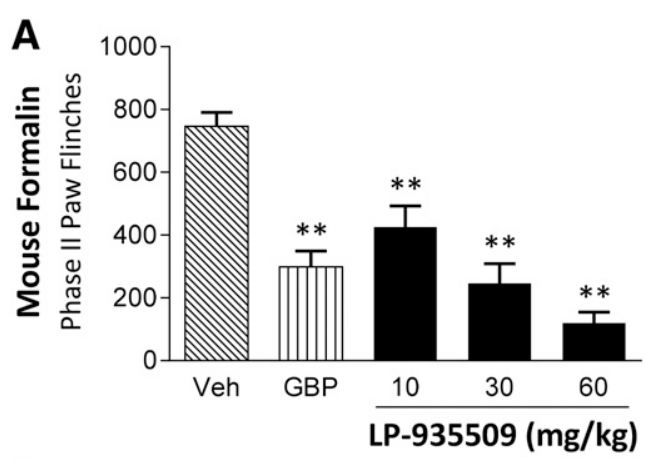

B
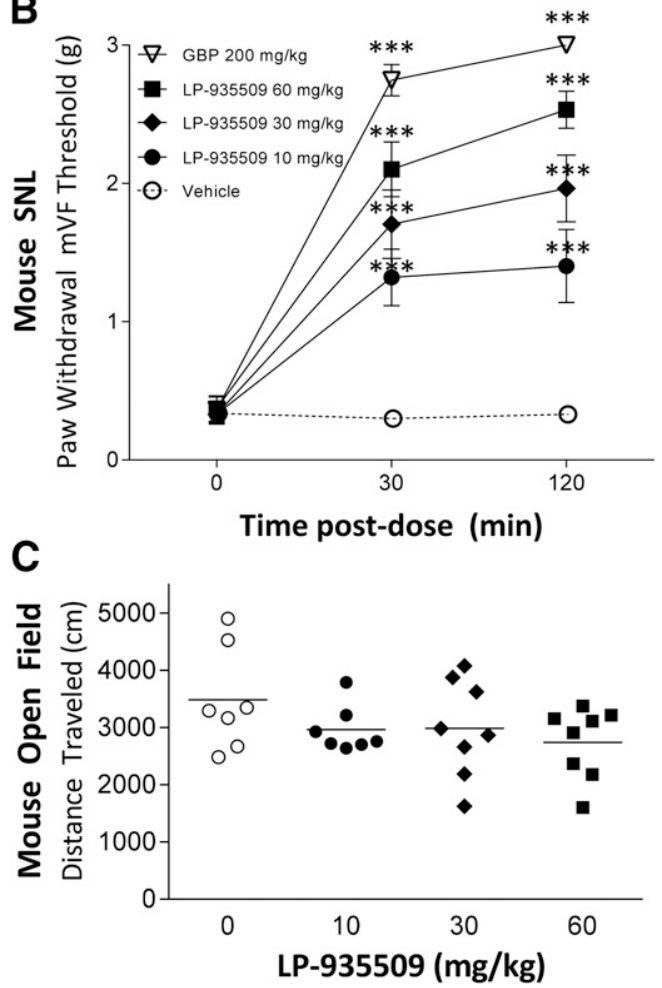

Fig. 3. AAK1 inhibitor LP-935509 recapitulates the knockout phenotype in mice. (A) Mouse phase II formalin responses after oral vehicle, gabapentin (GBP) $(200 \mathrm{mg} / \mathrm{kg})$, or LP-935509 $(10,30$, or $60 \mathrm{mg} / \mathrm{kg})(n=$ 8-10 male mice per group). $* * P<0.01$ versus Veh by one-way analysis of variance, followed by Dunnett's $t$ test. (B) Mechanical allodynia over time postdose [doses as in (A)] in mice with SNL injury after oral vehicle, GBP, or LP-935509 ( $n=9-13$ male mice per group). $* * * P<0.001$ versus vehicle by repeated measures analysis of variance, followed by Dunnett's $t$ test. (C) Open field locomotor activity of mice at 30 minutes after oral [doses as in (A)] vehicle, GBP, or LP-935509 ( $n=7$ male mice per group). $P=0.27$ by one-way analysis of variance.

with drug levels with efficacy delayed approximately $\sim 2-3$ hours from exposure.

LP-935509 was then evaluated in a 5-day repeat dosing study in SNL mice to test for tolerance (Fig. 5). Mice received two daily doses of 3,10 , or $30 \mathrm{mg} / \mathrm{kg}$ per day for 5 days. Efficacy was consistent at each dose level from day to day, revealing no evidence for tolerance or potentiation with repeated dosing. Exposures and efficacy levels were consistent with previous studies. Plasma exposures indicated compound levels were not altered by repeated dosing relative to single doses (Supplemental Table 6).

Given the potent inhibition of BIKE activity and the absence of data regarding the impact of BIKE inhibition on

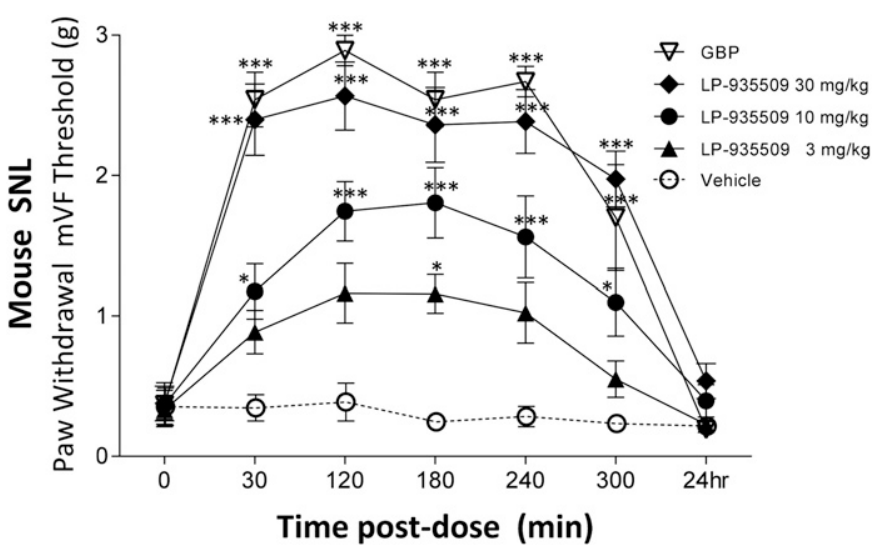

Fig. 4. AAK1 inhibitor LP-935509 efficacy in SNL model follows plasma exposure. Mouse SNL mechanical allodynia responses after oral vehicle, gabapentin (GBP) $(200 \mathrm{mg} / \mathrm{kg})$, or LP-935509 $(3,10$, or $30 \mathrm{mg} / \mathrm{kg})(n=4-7$ male mice per group). $* P<0.05$ and $* * * P<0.001$ versus vehicle by repeated measures analysis of variance, followed by Dunnett's $t$ test.

neuropathic pain, we generated BIKE knockout mice. SNL surgery of BIKE knockout mice revealed that these mice developed 30\% less allodynia after SNL surgery than wildtype mice (Supplemental Fig. 3). Although the loss of BIKE was not as impactful as AAK1 loss on mechanical allodynia in the SNL model, BIKE inhibition may contribute to antinociception from LP-935509.

An AAK1 Inhibitor Is Antinociceptive in Rat Models of Neuropathic Pain but Not Acute Pain. To facilitate the use of LP-935509 in rat behavioral studies, the pharmacokinetic properties of LP-935509 in rats were quantified. For these studies, three rats were dosed i.v. with $2 \mathrm{mg} / \mathrm{kg}$ LP-935509 and three rats were dosed orally with $10 \mathrm{mg} / \mathrm{kg}$ LP-935509. Blood samples were taken 10 times over a 24-hour period, and LP-935509 plasma levels were determined (Supplemental Fig. 5). Pharmacokinetic parameters were determined by noncompartmental analysis. This analysis showed that LP-935509 had 50\% oral bioavailability and a plasma half life of 4.0 hours. Brain drug levels exceeded plasma drug levels, with a brain/plasma drug ratio of 2.3 showing that LP-935509 was highly brain-penetrant. The Cmax for the $10 \mathrm{mg} / \mathrm{kg}$ oral dose was $3.1 \mu \mathrm{M}$ at 1.3 hours postdose. In addition, LP-935509 had a plasma-free fraction of $2.6 \%$ in rat. Based on these data, LP-935509 was considered a suitable compound for oral delivery to evaluate the effect of AAK1 inhibition on nociception.

The effect of LP-935509 on antinociception was further explored in rats because this species is commonly used to evaluate novel antinociceptive agents. LP-935509 was first tested in the CCI model. In this assay, the sciatic nerve is loosely ligated, which creates a neuropathic state within a few days after surgery, which lasts for several months (Bennett and Xie, 1988). In CCI animals, oral LP-935509 caused a dose-dependent reversal of thermal hyperalgesia (Fig. 6A), cold allodynia (Fig. 6B), mechanical allodynia (Fig. 6C), and mechanical hyperalgesia (Fig. 6D). In particular, LP-935509 completely reversed thermal hyperalgesia and mechanical allodynia at 10 and $30 \mathrm{mg} / \mathrm{kg}$, with most assays showing significant antinociceptive activity at lower doses (ranging from $0.3 \mathrm{mg} / \mathrm{kg}$ to $3 \mathrm{mg} / \mathrm{kg}$ ). The maximal reversal of the pain response was similar to that obtained by a high-dose gabapentin. The effects of LP-935509 on the CCI pain 


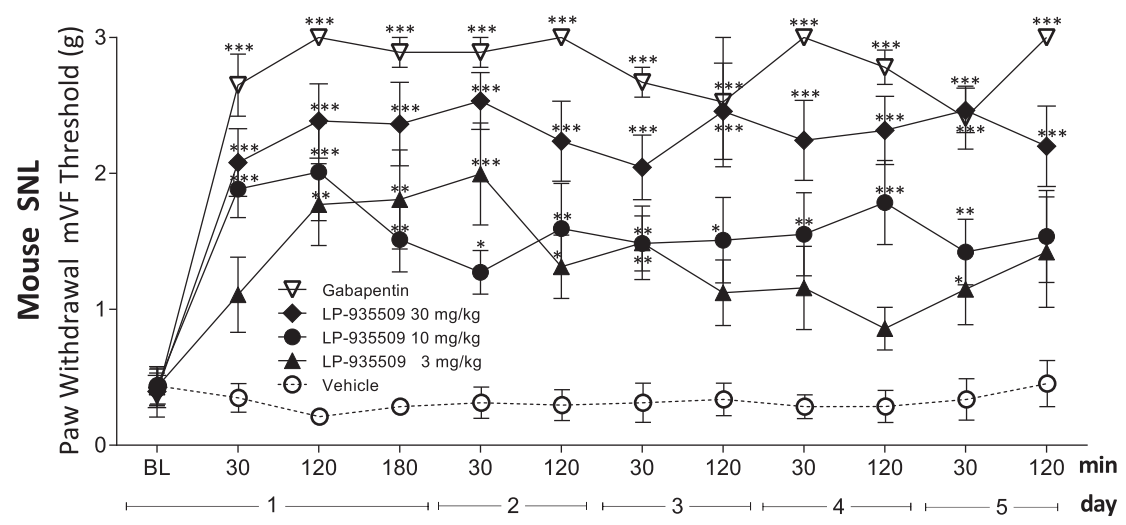

Time
Fig. 5. AAK1 inhibitor LP-935509 efficacy in SNL model does not change with repeat dosing. Mouse SNL mechanical allodynia responses with twice-daily dosing over 5 consecutive days with oral vehicle, gabapentin $(200 \mathrm{mg} / \mathrm{kg})$, or LP-935509 $(3,10$, or $30 \mathrm{mg} / \mathrm{kg})$ ( $n=4-7$ male mice per group). $* P<0.05,{ }^{*} P<0.01$, and $* * * P<0.001$ versus vehicle by repeated measures analysis of variance, followed by Dunnett's $t$ test. responses were not due to reduced functional motor abilities as oral LP-935509-treated animals were indistinguishable from vehicle-treated animals in the rat accelerating rotarod assay (Fig. 6E). In contrast, the $100 \mathrm{mg} / \mathrm{kg}$ dose of gabapentin used in these studies caused motoric impairment, which could confound measurement of the pain response. In our studies, gabapentin effects on pain behavioral measurements and impairment in rat rotarod were not well separated, with impairment in rotarod occurring at doses as low as $30 \mathrm{mg} / \mathrm{kg}$, the minimal efficacious dose (Supplemental Fig. 6).

LP-935509 was then tested in the STZ model of diabetic peripheral neuropathy (Courteix et al., 1993). In this model, rats are administered STZ (50 $\mathrm{mg} / \mathrm{kg})$, which kills insulinproducing pancreatic $\beta$ islet cells, thereby creating a diabetic state that is accompanied by allodynia and hyperalgesia, which increase progressively over 2-4 weeks. LP-935509 caused a dose-dependent reversal of mechanical allodynia in the STZ-treated rats to control levels (Fig. 6F). The antinociceptive efficacy observed at $3 \mathrm{mg} / \mathrm{kg}$ to $30 \mathrm{mg} / \mathrm{kg}$ was similar to high-dose $(100 \mathrm{mg} / \mathrm{kg})$ gabapentin.

LP-935509 plasma and brain drug levels were measured (Supplemental Table 3) after the behavioral studies in CCI and STZ rats (Fig. 6, A-F). In these studies, LP-935509 reversed the behavioral deficits, with $\mathrm{ED}_{50}$ values ranging from $2 \mathrm{mg} / \mathrm{kg}$ to $10 \mathrm{mg} / \mathrm{kg}$, which corresponded to plasma drug levels at 180-240 minutes that were about eightfold higher than the cellular $\mathrm{IC}_{50}$ value of $2.7 \mathrm{nM}$ (Supplemental Table 3).

LP-935509 was then tested in assays for acute pain, including the hot-plate and tail-flick assays. In the hot-plate assay, animals are placed in a chamber on a hot $\left(55^{\circ} \mathrm{C}\right)$ temperature-controlled surface, and the time to exhibit a hind limb response (e.g., licking or dancing) or a jump-escape response (i.e., leaping up onto the top edge of the enclosing chamber) is recorded (Woolfe and MacDonald, 1944). The time between placement of the animal into the test chamber and the display of a response is recorded as the hind limb response latency. To prevent tissue damage, animals that fail to exhibit a hind limb response by the cutoff time of 30 seconds are removed from the test chamber. In the tail-flick assay, a radiant heat source is positioned above the tail and directed through an aperture to stimulate the tail (D'Amour and Smith, 1941). The time between lamp onset and the response of flicking the tail to escape the thermal stimulus is recorded as tail-flick latency. To prevent tissue damage, animals that fail to respond to the radiant heat by the cutoff time of 10 seconds are removed from the stimulus. In both the hot-plate and tail-flick assays, $30 \mathrm{mg} / \mathrm{kg}$ oral LP-935509 did not affect the pain response, whereas $5 \mathrm{mg} / \mathrm{kg}$ morphine was highly analgesic (Fig. 6, G and H). The $30 \mathrm{mg} / \mathrm{kg}$ LP-935509 dose is maximally efficacious in our neuropathic pain models. Taken together, these results show that LP-935509 reduces neuropathic pain behaviors in mice and rats but does not affect acute pain perception.

AAK1 Inhibitors Cause Antinociception by Inhibiting AAK1 in the Spinal Cord. AAK1 is broadly distributed throughout the body, and LP-935509 is a highly permeable compound that is widely distributed to tissues. Hence, neither the target location nor the compound distribution is informative as to the location of the relevant pool of AAK1 for antinociception. To help address this question, LP-922761 was identified (Fig. 2C). LP-922761 is a potent and selective AAK1 inhibitor with an in vitro $\mathrm{IC}_{50}$ value of $4.8 \pm 0.8 \mathrm{nM}$ and a cellular $\mathrm{IC}_{50}$ value of $7.6 \pm 0.7 \mathrm{nM}$ (Table 1 ). Whereas LP-935509 has a brain to plasma drug level ratio $>2$, LP-922761 had a brain to plasma ratio of 0.007 , indicating that LP-922761 was essentially restricted to the peripheral compartment. When oral LP-927761 (60 mg/kg) was tested in the mouse for relief of mechanical allodynia following SNL surgery, the compound was not antinociceptive (Fig. 7A), whereas gabapentin $(200 \mathrm{mg} / \mathrm{kg})$ showed significant activity in the same assay. The inactivity of oral LP-922761 was not due to inadequate drug exposure because drug levels at the time of behavioral testing exceeded those of LP-935509 both with respect to total $(>11 \mu \mathrm{M})$ and free drug levels $(>1200$ nM) (Supplemental Table 3). These results with LP-922761 show that AAK1 inhibitors must cross the blood-brain barrier to be antinociceptive.

We next investigated intrathecal injection of an AAK1 inhibitor to determine whether AAK1 inhibitors could act at the level of the spinal cord. LP-935509 was not suitable for these studies due to poor aqueous solubility. Instead, we identified BMT-090605 (Fig. 2B) as a potent, selective AAK1 inhibitor (cellular $\mathrm{IC}_{50}=0.63+0.39 \mathrm{nM}$ ) with suitable physiochemical properties for intrathecal injection (Table 1). BMT-090605 (0.3-3 $\mu \mathrm{g} / \mathrm{rat})$ was delivered intrathecally at lumbar level L5/L6 to CCI animals. Behavioral evaluation of these animals showed that spinal delivery of BMT-090605 caused a dose-dependent reduction in thermal hyperalgesia (Fig. 7B). The high dose of BMT-090605 (3 $\mu \mathrm{g} / \mathrm{rat})$ showed efficacy comparable to intrathecal clonidine ( $3 \mu \mathrm{g} / \mathrm{rat})$. Exposure measurements from this study indicated that brain and plasma levels of BMT-090605 were $<4 \mathrm{nM}$, whereas 
A
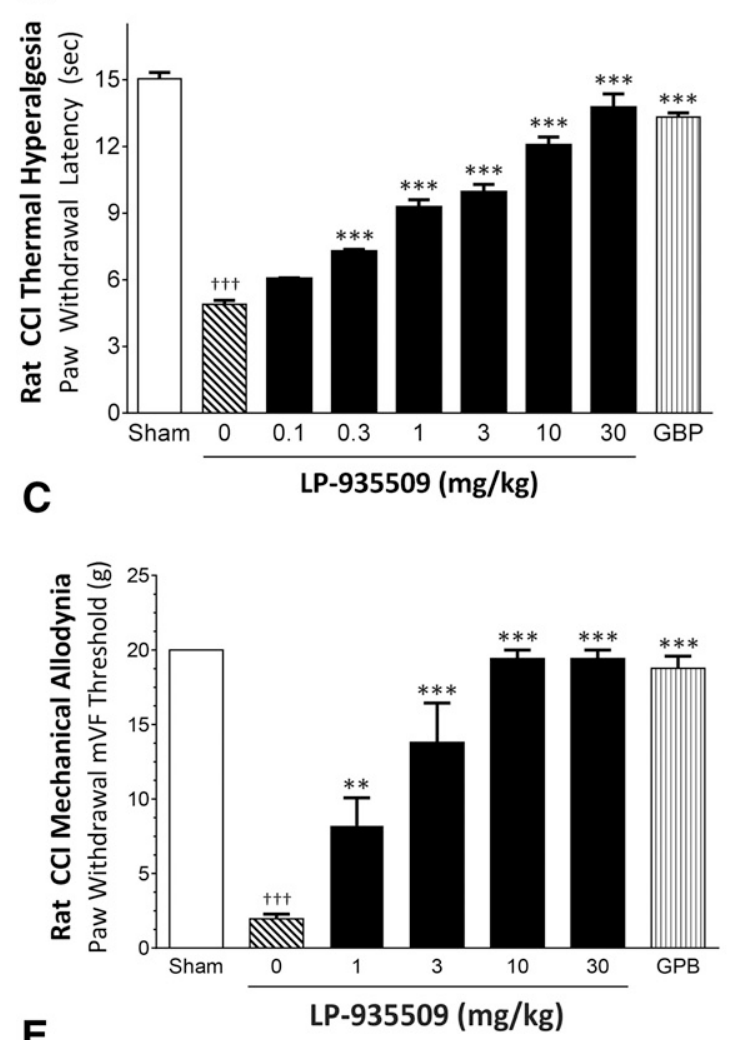

E

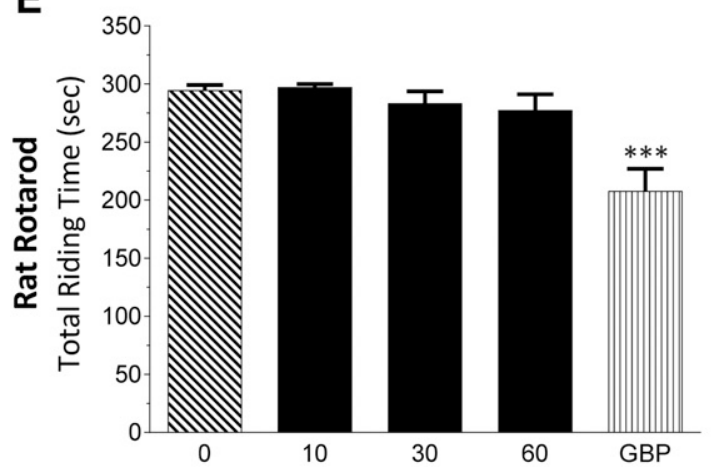

G

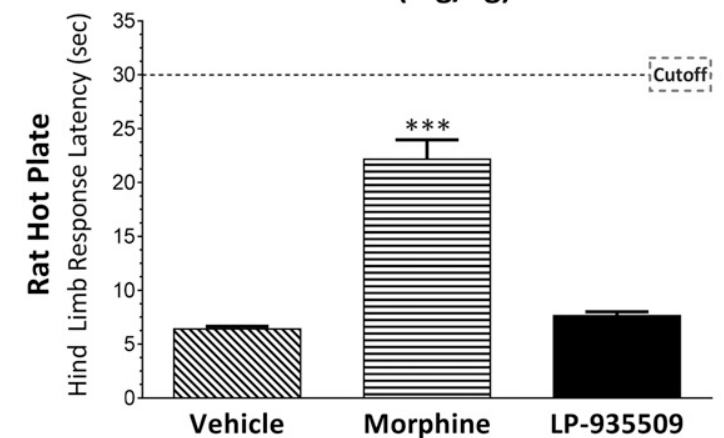

B
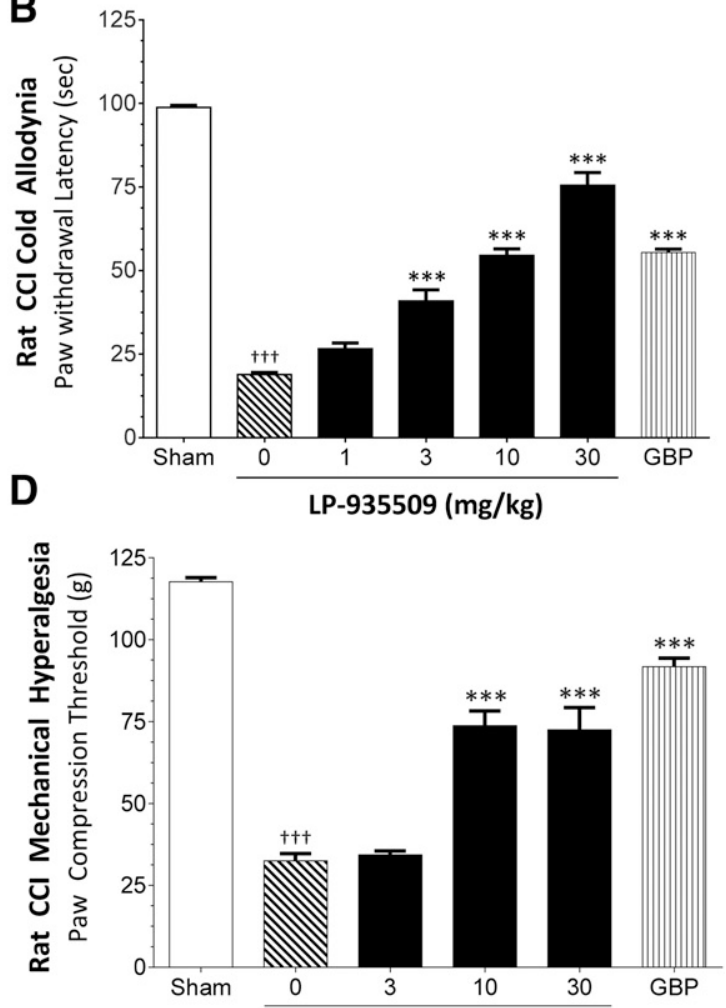

$\mathbf{F}$
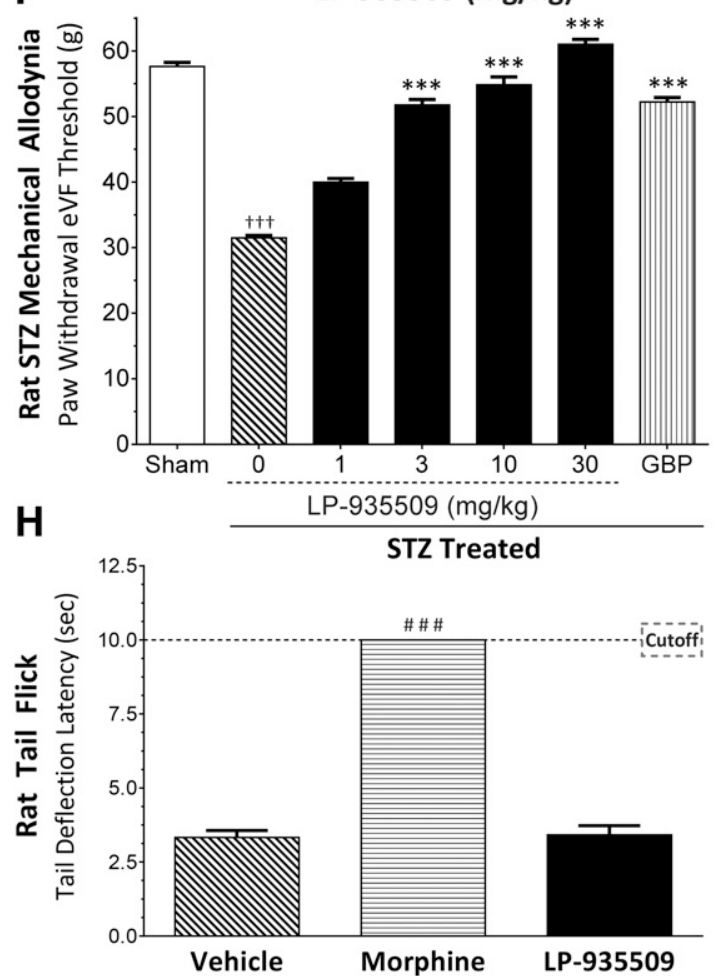

Fig. 6. AAK1 inhibitor LP-935509 is antinociceptive in multiple rat models of neuropathic pain, but not acute pain (A-D). CCI-operated rats were tested at 3 hours after oral vehicle (0), LP-935509 (at indicated doses), or gabapentin (GBP; $100 \mathrm{mg} / \mathrm{kg}$ ) in assays of (A) thermal hyperalgesia, (B) cold allodynia, (C) mechanical allodynia using Manual von Frey, or (D) mechanical hyperalgesia ( $n=7-8$ male rats per group). For comparison, sham-operated animals (Sham) are included. (E) Naive rats were testing in the accelerating rotarod assay, dosed as in Fig. 4, $(n=5-10$ male rats per group). (F) STZ-injured rats were tested for mechanical allodynia, dosed as in Fig. 4, $(n=7-8$ male rats per group). For comparison, sham-injected animals (Sham) are included. $(\mathrm{G}$ and $\mathrm{H})$ Naive rats were tested in assays of acute pain, including the following: $(\mathrm{G})$ hot plate and $(\mathrm{H})$ tail flick at $1.5 \mathrm{hours}$ after LP-935509 (30 mg/kg, Per Os po) or 0.5 hour after morphine ( $5 \mathrm{mg} / \mathrm{kg}$, s.c.) $(n=7-8$ male rats per group). $* * * P<0.001$ versus vehicle, or $\uparrow \dagger \dagger P<0.001$ versus sham controls by one-way analysis of variance, followed by Bonferroni's post hoc test; ${ }^{\# \#} P<0.001$ versus vehicle by paired $t$ test. 


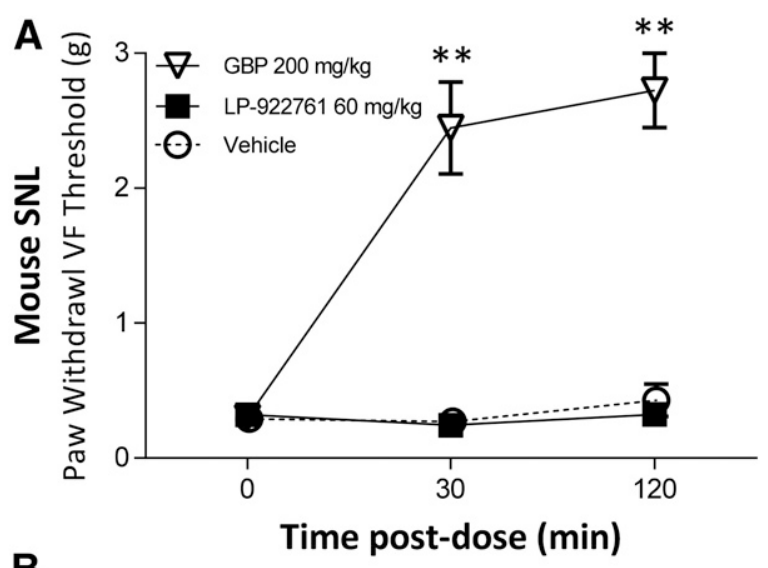

B

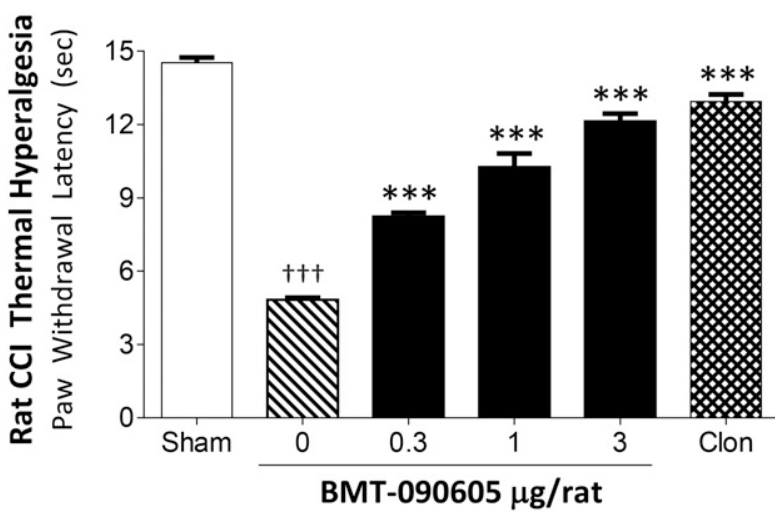

Fig. 7. AAK1 inhibitors cause antinociception by inhibiting AAK1 in the spinal cord. (A) Mice with SNL injury were tested for thermal hyperalgesia 2 hours after oral vehicle, LP-922761 (poorly brain-penetrant AAK1 inhibitor), or gabapentin (GBP) at indicated doses $(n=7-8$ male rats per group). (B) Rats with CCI surgery were tested for thermal hyperalgesia 15 minutes after intrathecal vehicle (0), BMT-090605 (0.3-3 $\mu \mathrm{g} / \mathrm{rat})$, or clonidine (Clon, $3 \mu \mathrm{g}$ ) ( $n=7-8$ male rats per group). For comparison, shamoperated animals (Sham) are included. $* * P<0.01 * * * P<0.001$ versus vehicle/ $0, \uparrow \dagger+P<0.001$ versus Sham by one-way analysis of variance, followed by Bonferroni's post hoc test.

measurable levels (90-317 nM) were observed in lumbar spinal cord (Supplemental Table 4). Total compound levels are $>100$-fold the cellular $\mathrm{IC}_{50}$ (Supplemental Table 3 ) at the lowest dose. In addition, there is most likely a compound gradient in the tissue with higher exposures in the painrelated dorsal horn. These data show that AAK1 inhibition localized to the spinal cord is sufficient for antinociceptive efficacy in CCI rats.

AAK1 Inhibitors Decrease Neural Activity in PainRelated Circuits at the Spinal Level. Rats with chronic constriction injury have increased spontaneous activity in the dorsal horn neurons of the spinal cord (Laird and Bennett, 1993; Liu and Walker, 2006). To test the effect of AAK1 inhibitors on this activity, we developed methods to record spontaneous activities from the spinal cord in anesthetized rats. In this assay, spontaneous action potential spikes from single neurons in the lamina $\mathrm{V}$ of the spinal cord were isolated and recorded extracellularly, and the response of the spike frequency to compound treatment was tested. These studies showed that rats that had undergone the CCI procedure had elevated spontaneous activity at an average of $23.3 \pm 2.4 \mathrm{~Hz}$ in the spinal dorsal horn neurons in comparison with $1.2 \pm$ $0.3 \mathrm{~Hz}$ in rats that had not undergone the CCI procedure.
The effect of LP-935509 on this increased spontaneous activity was then tested using i.v. delivery to more precisely control the onset of drug action upon electrophysiological responses. These studies showed that LP-935509 reduced CCI-induced increases in spontaneous activity at $3 \mathrm{mg} / \mathrm{kg}$ (Fig. 8A). LP-935509 effects on spontaneous firing began within 8 minutes of infusion and achieved near-complete blockade of injury-induced activity by 10 minutes. Blockade persisted for another 10 minutes and then showed a partial reversal at later times consistent with an expected reduction in drug levels. These studies were extended to multiple doses showing that LP-935509 (0.3-3 mg/kg) caused a dose-dependent reduction in spontaneous activity in CCI animals (Fig. 8B). LP-935509 was effective in the majority of neurons (7 of 10) in this study, suggesting that AAK1 antinociception is restricted to a subset of neurons that are activated by CCI. To determine whether AAK1 antinociception required the engagement of descending pain pathways, the spinal cord of CCI rats was transected at T8-T9, and the effect of LP-935509 on spontaneous activity was tested. AAK1 inhibition continued to produce a suppression of injury-induced activity in spinally transected animals and also achieved a near-complete blockade lasting for several minutes (Fig. 8C). Comparing these two experiments showed that spinal cord transection did not affect the AAK1 inhibition-induced reduction of spontaneous activity in CCI rats. The effect of LP-935509 (1 mg/kg) was the same regardless of whether the spinal cord was intact (Fig. 6B) or transected (Fig. 8D). These data indicate that intact descending pathways are not required for AAK1 inhibitor effects on spontaneous firing and suggest that either peripheral or local spinal action is sufficient to account for the observed reduction in spontaneous activity in CCI rats.

Windup is another electrophysiologic measure that is affected by drugs that are used to treat neuropathic pain (Arendt-Nielsen et al., 2011). In the animal assay of windup, a train of 16 electrical stimuli $(2 \mathrm{~ms}, 0.5 \mathrm{~Hz}, 2 \mathrm{~mA})$ was delivered transcutaneously to the hind paw to activate C-fiber-mediated windup activity of the wide-dynamic range neurons in the spinal dorsal horn of naive rats, and the effect of LP-935509 on the windup activity was then examined. These results showed that 1 and $3 \mathrm{mg} / \mathrm{kg}$ LP-935509 administered i.v. reduced windup activity in a dose-dependent manner (Fig. $8, \mathrm{E}$ and $\mathrm{F}$ ). In this study, 9 of 12 animals significantly responded to LP-935509, suggesting that a majority, but not all, neurons in the pain circuit are regulated by AAK1. To determine whether the AAK1 inhibitor effects on windup were operating in the spinal cord, BMT-124110 (cellular $\mathrm{IC}_{50}=3.2$ $+1.4 \mathrm{nM}$ ) (Fig. 2D) was added directly onto the spinal cord by microiontophoresis, leading to reduction in windup-induced activity in 10 of 14 animals (Fig. 8, G and H). BMT-124110 is structurally similar to BMT-090605, but had the appropriate combination of solubility and charge required for microiontophoresis. In this procedure, double-barrel microelectrodes were used to record neuronal activity and to apply BMT124110 by microiontophoresis. Taken together, these results indicate that AAK1 antinociception results from inhibition of pain circuits in the spinal cord.

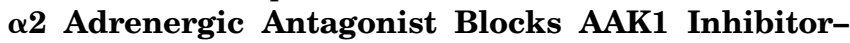
Induced Antinociception and Inhibition of Spontaneous Neural Activity. To further probe the mechanism of AAK1 inhibitor antinociception, chemical inhibitors of known pain pathways were tested to determine whether they blocked 


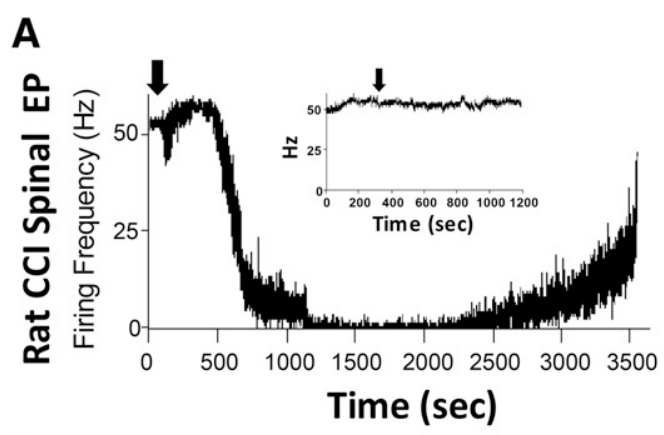

C

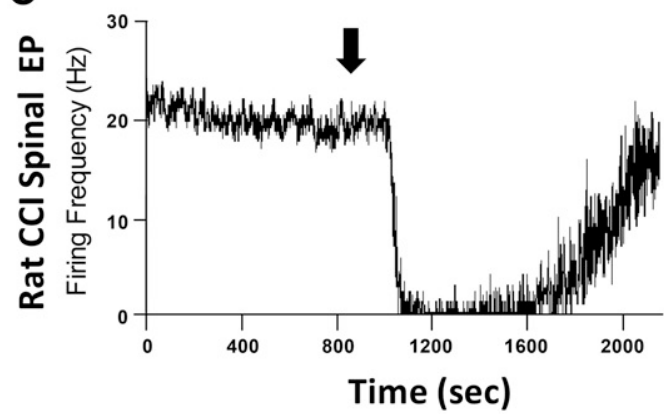

E

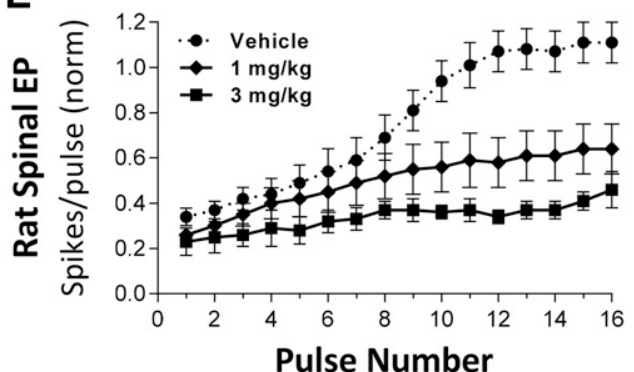

G

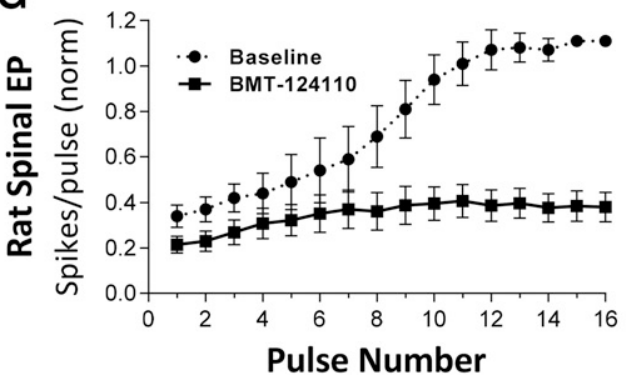

B

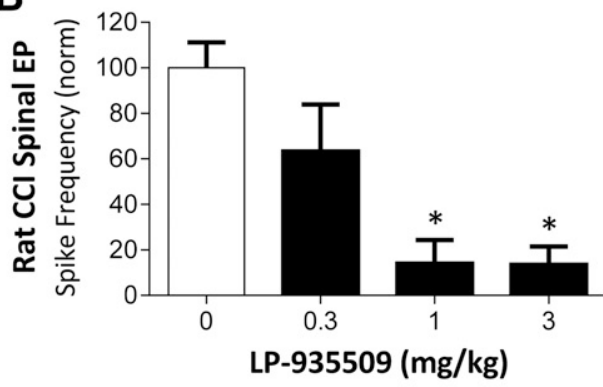

D

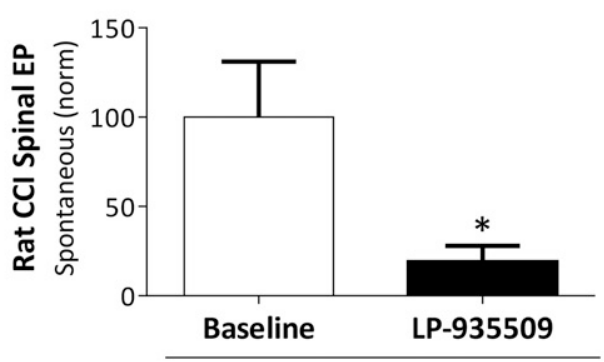

Spinal Transection at T8-T10

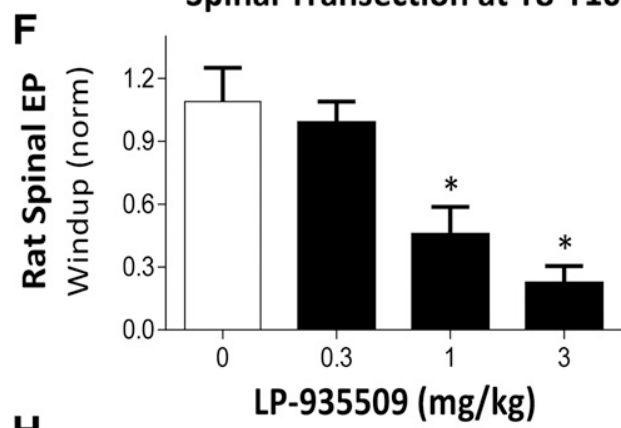

H

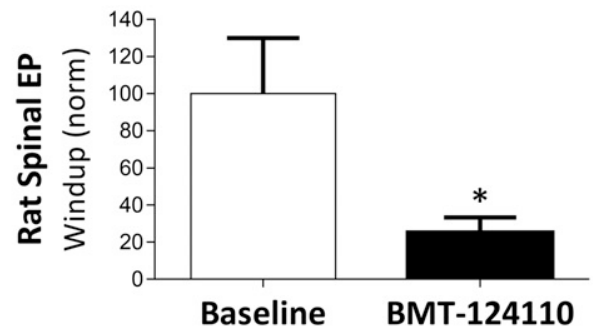

Fig. 8. AAK1 inhibitors decrease neural activity in pain-related circuits. (A) CCI rat spontaneous activity over time of a single spinal dorsal horn neuron showing inhibition by LP-935509 (1 mg/kg, i.v.). Arrow indicates time of LP-935509 injection (inset shows response to vehicle). (B) Dose-dependent inhibition by LP-935509 (0.3-3 mg/kg, i.v.) on spontaneous activity of spinal dorsal horn neurons in CCI male rats (responders, $n=3 / 4$ at $1 \mathrm{mg} / \mathrm{kg}, n=4 / 6$ at $3 \mathrm{mg} / \mathrm{kg}$ ). $* P<0.05$ versus vehicle (0) by one-way analysis of variance, followed by Dunnett's $t$ test. (C) Absence of effect of thoracic T8-T10 transection on LP-935509 (1 mg/kg, i.v.) inhibition of spontaneous activity over time in a single spinal dorsal horn neuron of a CCI male rat (arrow indicates time of injection). (D) Effect of LP-935509 (1 mg/kg, i.v.) on normalized spontaneous activity of CCI male rats $(n=4)$ with thoracic T8-10 transection. * $P<0.05$ versus baseline by unpaired $t$ test. (E) Naive wide-dynamic range neuron spikes evoked by hind limb repetitive electrical stimulation (16 pulses, 2 ms, 2.0 $\mathrm{mA}$ ) with effect of systemic vehicle or LP-935509 (1-3 mg/kg, i.v.) across pulses $(n=4-8$ male rats per group). (F) Systemic inhibition by LP-935509 $(0.3-3 \mathrm{mg} / \mathrm{kg}$, i.v.) of electrically evoked windup in spinal wide-dynamic range neurons from naive male rats (responders, $n=5 / 8$ at $1 \mathrm{mg} / \mathrm{kg}, n=4 / 4 \mathrm{at}$ $3 \mathrm{mg} / \mathrm{kg}$ ). $* P<0.05$ versus vehicle (0) by one-way analysis of variance, followed by Dunnett's $t$ test. (G) Naive rat spinal wide-dynamic range neuron spikes across pulses (as in Fig. 6E) with effect of microiontophoretically-applied BMT-124110 (30 mM, pH 4, $+10-30$ nA, 3 minutes) $(n=10$ male rats per group). (H) Spinal inhibition by BMT-124110 (as in Fig. 6G) of electrically evoked windup in dorsal horn wide-dynamic range neurons from naive male rats (responders, $n=10 / 14)$. $* P<0.05$ by unpaired $t$ test.

AAK1 inhibitor effects. We first tested the effect of naloxone, an inhibitor of opioid signaling. These studies showed that naloxone did not affect the ability of LP-935509 to reduce mechanical allodynia in the STZ model of diabetic pain (Fig. 9A). Naloxone was pharmacologically active in these experiments because naloxone fully inhibited the activity of the opioid agonist morphine. These data indicate that AAK1 inhibitors do not work through or require opioid signaling. In a separate study, we evaluated potential interactions with the noradrenergic painregulating system. Yohimbine, an inhibitor of $\alpha 2$ adrenergic 


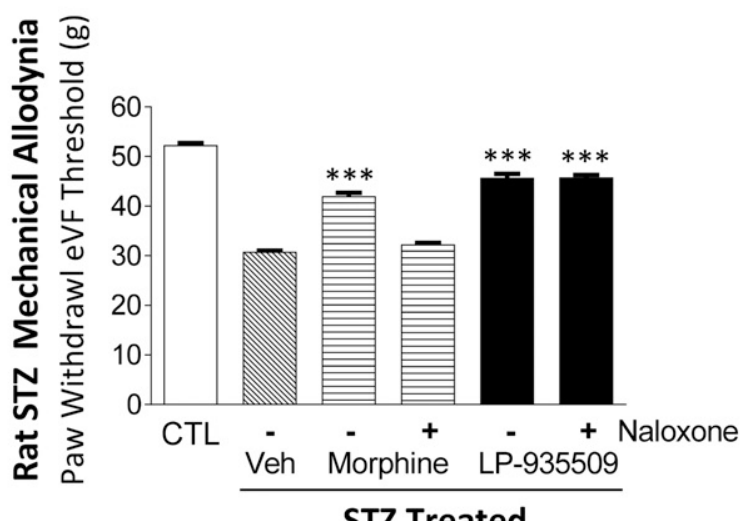

B

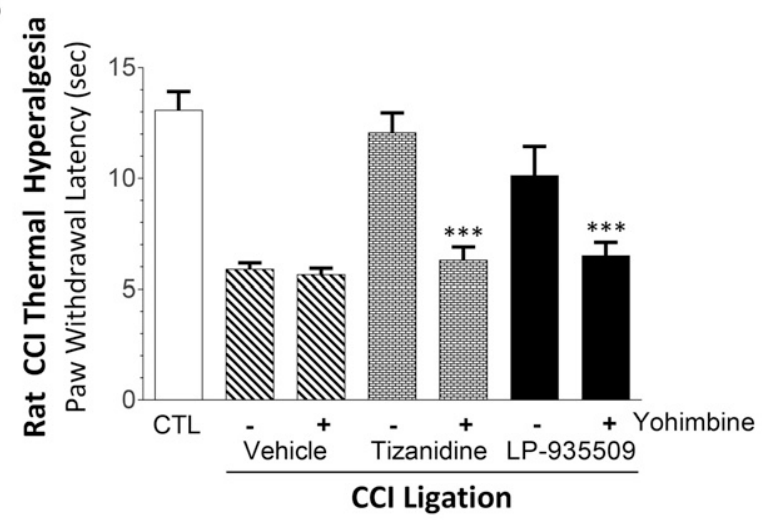

C

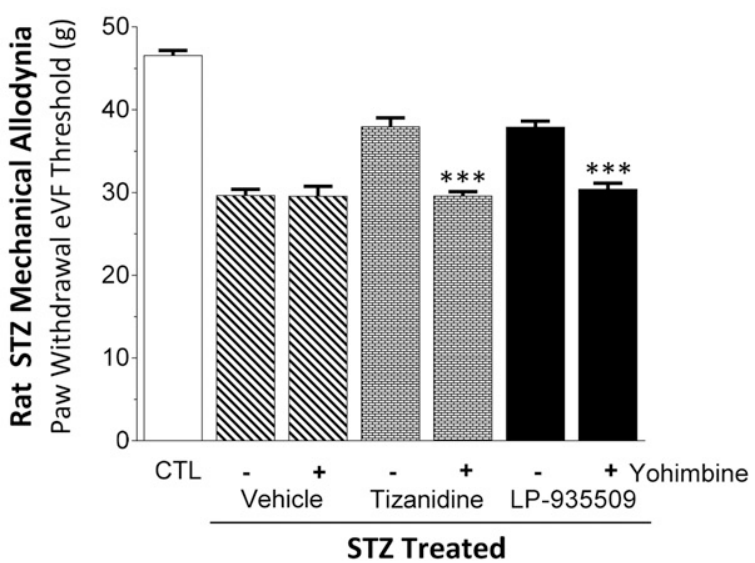

Fig. 9. $\alpha 2$ adrenergic antagonist blocks AAK1 inhibition-induced antinociception in CCI and STZ rats. (A) STZ-treated rats were tested for mechanical allodynia using electronic von Frey 1.5 hours after vehicle (per os), LP-935509 (30 mg/kg, per os), or morphine (3 mg/kg, s.c.) delivered in the presence $(+)$ or absence $(-)$ of naloxone $(1 \mathrm{mg} / \mathrm{kg}$, s.c.) given 30 minutes prior to the other agents ( $n=8$ male rats per group). For comparison, sham-treated (CTL) animals are included. *** $P<0.001$ versus vehicle by one-way analysis of variance, followed by Bonferroni's post hoc test. (B) CCI-operated rats were tested for thermal hyperalgesia 30 minutes after tizanidine $(1 \mathrm{mg} / \mathrm{kg}$, i.p.), or 1.5 hours after either vehicle (per os) or LP-935509 (30 mg/kg, po) all delivered in the presence $(+)$ or absence $(-)$ of yohimbine $(1 \mathrm{mg} / \mathrm{kg}$, i.p.) given 100 minutes before testing $(n=6-11$ male rats per group). CTL as in (A). ${ }^{* * *} P<0.001$ versus $(-)$ yohimbine pair by one-way analysis of variance, followed by Bonferroni's post hoc test. (C) STZ-treated rats were tested for mechanical allodynia with CTL and dosing as in (B) $\left(n=8\right.$ male rats per group). ${ }^{* * *} P<0.001$ versus $(-)$ yohimbine as in (B).

receptors, prevented LP-935509 reductions in CCI thermal hyperalgesia and STZ mechanical allodynia (Fig. 9, B and C). As expected, yohimbine also blocked the antinociceptive effects of tizanidine, an $\alpha 2$ adrenergic agonist, in these thermal and mechanical hyperalgesia assays. LP-935509 exposures did not differ significantly with and without yohimbine cotreatment (Supplemental Table 5). Similar data were generated with the AAK1 inhibitor BMT-090605 (data not shown), indicating the interaction of AAK1 and $\alpha 2$ adrenergic pathways was a general feature of AAK1 inhibition. LP-935509 and BMT-090605 do not alter adrenergic ligand binding to human $\alpha 2 \mathrm{~A}$ and $\alpha 2 \mathrm{C}$ receptors and showed little or no agonist activity in recombinant cellular $\alpha 2 \mathrm{~A}$ and $\alpha 2 \mathrm{C}$ cellular assays (Table 1 ). These data suggest that these AAK1 inhibitors are not acting directly on $\alpha 2$ adrenergic receptors.

Similar pharmacologic effects were also seen in wholeanimal in vivo electrophysiology studies of CCI rats. The LP-935509-induced reduction in CCI-evoked spontaneous activity (Fig. 10A, top trace) was blocked by pretreatment with yohimbine (Fig. 10A, bottom trace). Yohimbine alone was without significant effect, but fully prevented the reduction in spontaneous activity of spinal cord dorsal horn neurons shown by LP-935509 (Fig. 8B). Thus, in both behavioral (Fig. 9B) and electrophysiological (Fig. 10) assays, yohimbine pretreatment prevented the beneficial effects of LP-935509 in CCI rats.

\section{Discussion}

This study identified AAK1 as a novel target for antinociception using the formalin assay to screen knockout mice. The ability of AAK1 knockouts to significantly reduce formalin phase II responses was extended to show that AAK1 knockouts also fail to develop mechanical allodynia in the mouse SNL model of neuropathic pain. AAK1 knockout mice exhibited no other phenotypes in a broad phenotypic screen, suggesting AAK1 is a promising target for reducing neuropathic pain while avoiding other mechanism-based toxicities. Based on these findings, selective and potent small-molecule inhibitors of AAK1 kinase activity were discovered. These AAK1 inhibitors dose-dependently reduced nociceptive behavioral responses in multiple rodent assays of neuropathic pain, inhibited spontaneous neuronal activity in the spinal cord due to chronic constriction injury, and blocked the development of windup normally elicited by repeated electrical pulses. The effects in behavioral assays of neuropathic pain were present across a variety of stimulus modalities (thermal, mechanical, chemical, electrical) without confounding motor deficits. Multiple-day dosing of AAK1 inhibitor in the SNL model showed no evidence of tolerance.

AAK1 inhibition did not alter acute nociception in the phase I formalin test, hot-plate assay, and tail-flick assays, suggesting that AAK1 inhibition causes a selective antineuropathic effect while leaving normal nociception intact. The behavioral impact of AAK1 inhibitors was mirrored electrophysiologically in the selective dampening by AAK1 inhibition of spontaneous neuronal activity following CCI injury, while preserving some ongoing activity like that observed in noninjured control animals. Similarly, the peak effect of AAK1 inhibition observed in normal animals in the windup assay did not drop below the baseline activity observed in the first pulse.

The blockade of AAK1 inhibitor-induced antinociception and electrophysiologic effects by $\alpha 2$-adrenergic inhibitors links AAK1 inhibition to a major inhibitory pain pathway 

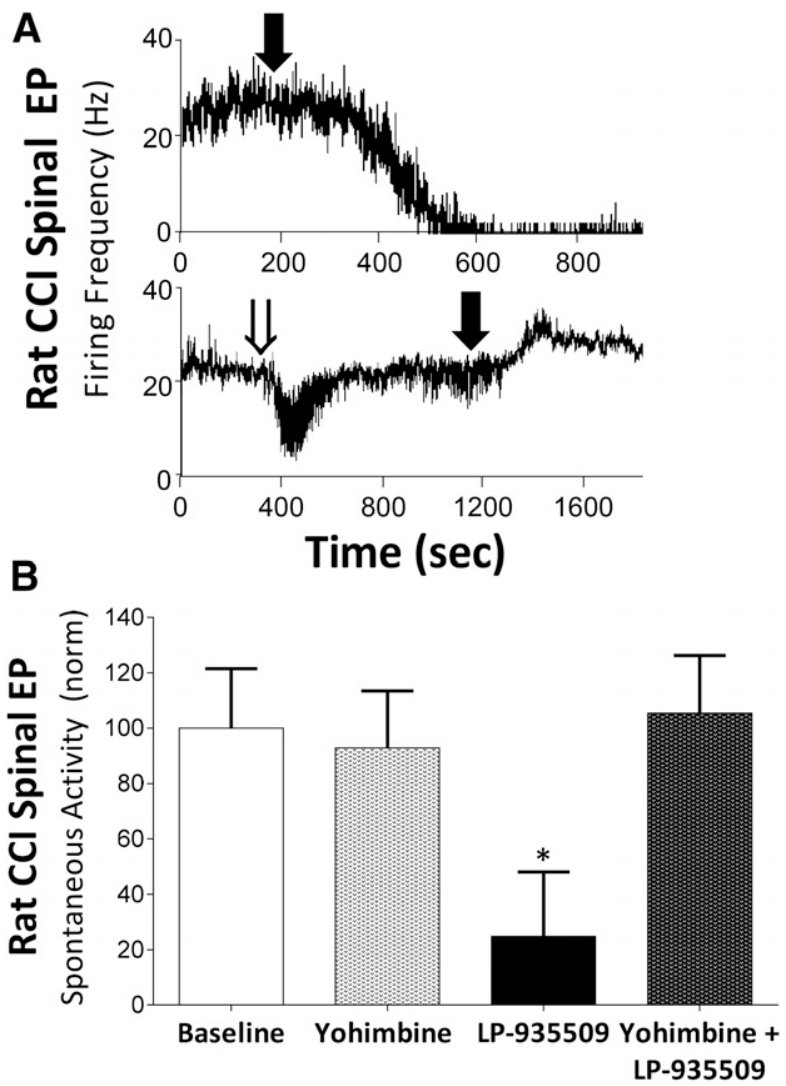

Fig. 10. $\alpha 2$ adrenergic antagonist blocks AAK1 inhibition-induced reduction in spontaneous neuronal activity in CCI rats. (A) CCI rat spontaneous activity time course of two individual spinal dorsal horn neurons treated with LP-935509 (1 mg/kg, i.v.; filled arrow) without (top trace) or with (bottom trace) pretreatment of yohimbine $(0.3 \mathrm{mg} / \mathrm{kg}$, i.v.; open arrow). (B) CCI rat group data for spontaneous activity with design and dosing as in (A) plus baseline and yohimbine controls $(n=6-8$ male rats per group). $* P<0.05$ versus baseline by one-way analysis of variance, followed by Dunnett's $t$ test.

used by endogenous and exogenous antinociceptives. In particular, the most commonly prescribed medications for neuropathic pain, gabapentinoids, tryclic antidepressants, and SNRIs, act (at least in part) through the $\alpha 2$-adrenergic pathway (Fairbanks et al., 2009). In addition to linking AAK1 to the $\alpha 2$-adrenergic pathway, our studies provide clues to the antinociceptive mechanism of AAK1 inhibitors. The inhibitory effects of AAK1 inhibitors in spinal cord preparations and failure of spinal transection to reduce AAK1 inhibitor suppression of spontaneous activity suggest that AAK1 inhibition acts at a spinal level to reduce ascending pain signals. This may involve modification of synaptic transmission from dorsal root ganglion cell terminals in the dorsal horn or even altering local circuits within the dorsal horn. Attempts to further define the cellular site of action using spinal slice electrophysiology were hampered by the paucity of tools to precisely define and modulate specific neuronal populations. AAK1 mRNA is expressed in the many brain areas as shown in the Allen Brain Atlas, and AAK1 protein immunoreactivity is detected in the spinal cord (Shi et al., 2014). Thus, although we have focused on the spinal cord site of action as sufficient for AAK1 inhibitor antinociceptive effects, we cannot rule out a possible supraspinal contribution with systemic administration.
Given the role for AAK1 in endocytosis, we hypothesized that reduced endocytosis of a key cell surface protein(s) involved in transmitting pain signals could explain AAK1 antinociception. Based on this hypothesis, we searched for proteins with the YXXPhi motif that might have a known function that could explain the role of AAK1 in neuropathic pain. Whereas neither $\alpha 2 \mathrm{~A}$ nor $\alpha 2 \mathrm{C}$ adrenergic receptors contained this motif, $\mathrm{GABA}_{\mathrm{A}} \gamma 2$ protein contained this motif. The GABA $\gamma 2$ protein is a component of $\mathrm{GABA}_{\mathrm{A}}$ channels that contain $\alpha, \beta$, and $\gamma$ subunits. Activation of these channels by GABA increases chloride conductance, thereby typically decreasing membrane potential and action potentials. The importance of endocytosis for regulating surface levels of $\mu 2$ containing $\mathrm{GABA}_{\mathrm{A}}$ channels is supported by two experiments. First, a peptide inhibitor of $\mathrm{GABA}_{\mathrm{A}} \mathrm{Cl}$ channel- $\mu 2$ binding increased $\mathrm{GABA}_{\mathrm{A}}$-dependent mini-inhibitory postsynaptic currents in striatal neurons (Kittler et al., 2008). Second, a naturally occurring $\mathrm{GABA}_{\mathrm{A}} \gamma 2$ protein mutation (R43Q) found in a patient with childhood epilepsy increased cell surface levels by decreasing endocytosis (Chaumont et al., 2013). The importance of $\mu 2$-dependent endocytosis on $\mathrm{GABA}_{\mathrm{A}} \mathrm{Cl}$ channel function is particularly interesting given the role of $\mathrm{GABA}_{\mathrm{A}} \mathrm{Cl}$ channel in nociception. In particular, multiple studies show that GABA agonists are antinociceptive in preclinical models of neuropathic pain but not human pain, perhaps due to dose-limiting sedation or tolerance (reviewed in McCarson and Enna, 2014; Zeilhofer et al., 2015). The antinociceptive activity of $\mathrm{GABA}_{\mathrm{A}}$ agonists has been localized to the spinal cord, where $\mathrm{GABA}_{\mathrm{A}}$ receptors are found on primary sensory neurons and intrinsic dorsal horn neurons. Taken together, the antinociceptive effects of increasing GABA activity and its dependence on $\mu 2$ endocytosis make $\mathrm{GABA}_{\mathrm{A}}$ channels an appealing hypothetical target of AAK1 antinociceptive effects.

Although we have focused on the more studied role of AAK1 in modulating cargo capture in clathrin-coated endocytosis through $\mu 2$ phosphorylation, there are several other AAK1related activities that have been reported. One group identified AAK1 (S635) as a substrate for the kinase NDR1/2 and showed that AAK1 plays a role in dendritic branching and length in cultured rat hippocampal neurons (Ultanir et al., 2012). The relevance of these observations for the role of AAK1 in neuropathic pain is unclear because these dendritic effects took days to manifest, whereas AAK1 antinociception occurred within minutes. Another group reported that AAK1 knockdown or inhibition leads to ErbB4 accumulation in PC12 cells and potentiates Nrg1-ErbB4-mediated neurite outgrowth (Kuai et al., 2011). Interestingly, intrathecal injection of the Nrg1 peptide was recently found to be antinociceptive in the SNL model (Wang et al., 2014). AAK1 has also been reported as a positive regulator of the notch pathway (GuptaRossi et al., 2011), which has been linked to pain. In particular, Notch inhibition with a $\gamma$-secretase inhibitor reduced neuropathic pain in the rat SNL model, whereas Jagged-1, a Notch ligand, increased pain sensitivity (Xie et al., 2015).

The failure of opioid antagonists to inhibit AAK1 inhibitor antinociception shows that AAK1 antinociception does not use the opioid system, a major inhibitory pain pathway used by endogenous and exogenous antinociceptives (Fields, 2004). Studies with $\alpha 2$ adrenergic agonists and opioids, however, raise the possibility that AAK1 inhibitors will synergize with opioids for antinociception. In particular, a major use 
for clonidine, an $\alpha 2$ adrenergic agonist, is as an opioid adjuvant. Synergy of $\alpha 2$ adrenergic agonists and opioids is supported by preclinical (Stone et al., 2014) and clinical studies (Teasell et al., 2010; Engelman and Marsala, 2013). AAK1 inhibition may provide a means to obtain this synergy with the ease of oral delivery while avoiding the unwanted side effects of systemic $\alpha$ adrenergic agonism.

The structural diversity of these AAK1 inhibitors used in this study supports the conclusion from AAK1 knockouts that AAK1 inhibition is antinociceptive. It was difficult to identify AAK1 inhibitiors that did not inhibit BIKE. Relatively little is known about BIKE; however, it has been implicated in the differentiation of osteoclasts in vitro (Kearns et al., 2001). We found that BIKE knockouts were normal in the phenotyping battery, except for partial resistance to pain in the SNL model, and bred normally, and BIKE-AAK1 double knockouts were viable and healthy (data not shown).

We observed minor differences in the predicted plasma-free drug coverage of the cellular $\mathrm{IC}_{50}$ values in mice relative to rats. In particular, efficacious free plasma concentrations for LP-935509 averaged 30-fold and eightfold the cellular $\mathrm{IC}_{50}$ in mice and rats, respectively. These differences were most likely due to the sampling times to determine drug levels in which mice were typically sampled 30-120 minutes postdose, whereas rats were typically sampled $180-240$ minutes postdose. Regardless of these differences, the comparison of free plasma drug levels with cellular $\mathrm{IC}_{50}$ values indicates that several fold coverage of the cellular $\mathrm{IC}_{50}$ is required for maximal antinociception.

The lack of significant nonpain-related phenotypes in the AAK1 knockout mice and AAK1 inhibitor-treated mice raises the hope that AAK1 inhibitors can provide antinociception without the side effects that limit current drugs targeting neuropathic pain. Gabapentinoids, such as gabapentin and pregabalin, which currently dominate the neuropathic pain market, are dose-limited by sedation and cognitive impairment and provide only very modest symptom control for most patients. We observe little or no separation between the minimum doses of gabapentin that produce efficacy in rat neuropathic pain models and those that cause impairment in the accelerating rotarod. Across the range of doses tested, those that produced significant efficacy also produced significant deficits in motor impairment. In contrast, in these same assays we have observed substantial separation between doses of AAK1 inhibitors that provide significant antinociceptive effect and the dose tested in motor impairment (which for AAK1 inhibitors failed to show any significant motor deficits at the maximum dose tested, suggesting potential for an even greater separation).

Using a large-scale mouse knockout and phenotypic screening approach, we identified AAK1 as a potential new target for neuropathic pain. We have further demonstrated that it is possible to generate brain-penetrant and orally active, selective AAK1 inhibitors with drug-like properties that are effective in both ligation and diabetic neuropathy models without dose-limiting side effects or alterations in normal nociception. We believe this represents a promising new direction for novel neuropathic pain therapy.

\section{Acknowledgments}

We thank Mian Gao, Yaqun Zhang, Brian Carpenter, Chunhong Yan, Frank Marsilo, John Newitt, and Nicholas Szapiel from BristolMyers Squibb (BMS) for expert production and purification of the recombinant kinases used in these studies; Kim Esposito and Ramesh Padmanabha of the BMS Lead Discovery and Optimization (LDO) Department for design and execution of the high throughput screen that produced the several important initial hits for the program; Shuli Wang of Lexicon for conducting a high throughput screen; Randi Brown of BMS LDO for expert execution of the AAK1 caliper assay; Jing Chen, Glen Farr, Michele Matchett, and Reshma Panemangalore of BMS LDO for receptor profiling in binding and functional assays; Krishna Pochá of BMS Biocon Research Center and Mike Donegan of BMS Preclinical Candidate Optimization (PCO) for analysis of biology and PK samples; and Ben Johnson of BMS PCO for skilled assessment of biotransformation. We acknowledge the chemistry efforts of SelvaKumar Bhaskaran, Tarun Maishal, Raju Manepalli, K.S. Maheswaran, Vivekananda Murthy Vrudhula, and Bireshwar Dasgupta.

\section{Authorship Contributions}

Participated in research design: Albright, Mandlekar, Vikramadithyan, Nara, Allen, Balakrishnan, Bristow, Brown, Carson, Denton, Dzierba, Bronson, Conway, Easton, Grace, Hamman, Kiss, Kostich, Lanthorn, Lentz, Li, Macor, Main, Nouraldeen, Santone, Savelieva, Baker, Swaffield, Westphal, Wilson, Ye, Zaczek, Zambrowicz.

Conducted experiments: Kumar, Das, Dokania, Shankar, Elavazhagan, Holenarsipur, Louis, Dandapani, Vattikundala, Sharma, Allen, Balakrishnan, Bi, Bourin, Brown, Feng, Grace, Gulianello, Hamman, Huang, Kiss, Lewis, Li, Lippy, Lu, Molski, Naidu, Nouraldeen, O’Malley, Pieschl, Savelieva, Baker, Ye.

Contributed new reagents or analytic tools: Hamman.

Performed data analysis: Allen, Balakrishnan, Bourin, Brown, Denton, Feng, Grace, Gulianello, Hamman, Kostich, Lentz, Li, Lippy, Nouraldeen, O'Malley, Savelieva, Baker, Swaffield, Westphal, Ye, Zambrowicz.

Wrote of contributed to the writing of the manuscript: Albright, Conway, Denton, Easton, Hamman, Kostich, Lentz, Li, Lippy, Savelieva, Baker, Zaczek, Zambrowicz.

\section{References}

Arendt-Nielsen L, Mansikka H, Staahl C, Rees H, Tan K, Smart TS, Monhemius R, Suzuki R, and Drewes AM (2011) A translational study of the effects of ketamine and pregabalin on temporal summation of experimental pain. Reg Anesth Pain Med 36:585-591.

Beltran del Rio H, Kern F, Lanthorn T, Oravecz T, Piggott J, Powell D, Ramirez-Solis R, Sands AT, and Zambrowicz B (2003) Saturation screening of the druggable mammalian genome, in Model Organisms in Drug Discovery (Carroll $\mathrm{P}$ and Fitzgerald K eds) pp 251-278, Wiley \& Sons, Chichester, UK.

Benarroch EE (2008) Descending monoaminergic pain modulation: bidirectional control and clinical relevance. Neurology 71:217-221.

Bennett GJ and Xie YK (1988) A peripheral mononeuropathy in rat that produces disorders of pain sensation like those seen in man. Pain 33:87-107.

Brommage R, Desai U, Revelli JP, Donoviel DB, Fontenot GK, Dacosta CM, Smith DD, Kirkpatrick LL, Coker KJ, and Donoviel MS, et al. (2008) High-throughput screening of mouse knockout lines identifies true lean and obese phenotypes. Obesity 16:2362-2367.

Brommage R, Liu J, Hansen GM, Kirkpatrick LL, Potter DG, Sands AT, Zambrowicz B, Powell DR, and Vogel P (2014) High-throughput screening of mouse gene knockouts identifies established and novel skeletal phenotypes. Bone Res 2:14034. Chaplan SR, Bach FW, Pogrel JW, Chung JM, and Yaksh TL (1994) Quantitative assessment of tactile allodynia in the rat paw. $J$ Neurosci Methods 53:55-63.

Chaumont S, André C, Perrais D, Boué-Grabot E, Taly A, and Garret M (2013) Agonist-dependent endocytosis of $\gamma$-aminobutyric acid type A (GABAA) receptors revealed by a $\gamma 2(\mathrm{R} 43 \mathrm{Q})$ epilepsy mutation. J Biol Chem 288:28254-28265.

Conner SD and Schmid SL (2002) Identification of an adaptor-associated kinase, AAK1, as a regulator of clathrin-mediated endocytosis. J Cell Biol 156:921-929.

Costigan M, Scholz J, and Woolf CJ (2009) Neuropathic pain: a maladaptive response of the nervous system to damage. Annu Rev Neurosci 32:1-32.

Courteix C, Eschalier A, and Lavarenne J (1993) Streptozocin-induced diabetic rats: behavioural evidence for a model of chronic pain. Pain 53:81-88.

D'Amour FE and Smith DL (1941) A method for determining loss of pain sensation. $J$ Pharmacol Exp Ther 72:74-79.

Dolphin AC (2012) Calcium channel auxiliary $\alpha 2 \delta$ and $\beta$ subunits: trafficking and one step beyond. Nat Rev Neurosci 13:542-555.

Dubuisson D and Dennis SG (1977) The formalin test: a quantitative study of the analgesic effects of morphine, meperidine, and brain stem stimulation in rats and cats. Pain 4:161-174.

Dunham NW and Miya TS (1957) A note on a simple apparatus for detecting neurological deficit in rats and mice. J Am Pharm Assoc Am Pharm Assoc 46:208-209.

Engelman E and Marsala C (2013) Efficacy of adding clonidine to intrathecal morphine in acute postoperative pain: meta-analysis. Br J Anaesth 110:21-27. 
Fairbanks CA (2003) Spinal delivery of analgesics in experimental models of pain and analgesia. Adv Drug Deliv Rev 55:1007-1041.

Fairbanks CA, Stone LS, and Wilcox GL (2009) Pharmacological profiles of alpha 2 adrenergic receptor agonists identified using genetically altered mice and isobolographic analysis. Pharmacol Ther 123:224-238.

Fields H (2004) State-dependent opioid control of pain. Nat Rev Neurosci 5:565-575.

Finnerup NB, Sindrup SH, and Jensen TS (2010) The evidence for pharmacological treatment of neuropathic pain. Pain 150:573-581.

Gupta-Rossi N, Ortica S, Meas-Yedid V, Heuss S, Moretti J, Olivo-Marin JC, and Israël A (2011) The adaptor-associated kinase 1, AAK1, is a positive regulator of the Notch pathway. J Biol Chem 286:18720-18730.

Hargreaves K, Dubner R, Brown F, Flores C, and Joris J (1988) A new and sensitive method for measuring thermal nociception in cutaneous hyperalgesia. Pain 32:77-88. Hayashida K, Obata H, Nakajima K, and Eisenach JC (2008) Gabapentin acts within the locus coeruleus to alleviate neuropathic pain. Anesthesiology 109:1077-1084.

Hayashida K, Parker R, and Eisenach JC (2007) Oral gabapentin activates spinal cholinergic circuits to reduce hypersensitivity after peripheral nerve injury and interacts synergistically with oral donepezil. Anesthesiology 106:1213-1219.

Hylden JL and Wilcox GL (1980) Intrathecal morphine in mice: a new technique. Eur $J$ Pharmacol 67:313-316.

Jackson AP, Flett A, Smythe C, Hufton L, Wettey FR, and Smythe E (2003) Clathrin promotes incorporation of cargo into coated pits by activation of the AP2 adaptor micro2 kinase. J Cell Biol 163:231-236.

Kearns AE, Donohue MM, Sanyal B, and Demay MB (2001) Cloning and characterization of a novel protein kinase that impairs osteoblast differentiation in vitro. $J$ Biol Chem 276:42213-42218.

Kim SH and Chung JM (1992) An experimental model for peripheral neuropathy produced by segmental spinal nerve ligation in the rat. Pain 50:355-363.

Kittler JT, Chen G, Kukhtina V, Vahedi-Faridi A, Gu Z, Tretter V, Smith KR, McAinsh K, Arancibia-Carcamo IL, and Saenger W, et al. (2008) Regulation of synaptic inhibition by phospho-dependent binding of the AP2 complex to a YECL motif in the GABAA receptor gamma2 subunit. Proc Natl Acad Sci USA 105 3616-3621.

Kuai L, Ong SE, Madison JM, Wang X, Duvall JR, Lewis TA, Luce CJ, Conner SD, Pearlman DA, and Wood JL, et al. (2011) AAK1 identified as an inhibitor of neuregulin-1/ErbB4-dependent neurotrophic factor signaling using integrative chemical genomics and proteomics. Chem Biol 18:891-906.

Laird JM and Bennett GJ (1993) An electrophysiological study of dorsal horn neurons in the spinal cord of rats with an experimental peripheral neuropathy. J Neurophysiol 69:2072-2085.

Liu C and Walker JM (2006) Effects of a cannabinoid agonist on spinal nociceptive neurons in a rodent model of neuropathic pain. J Neurophysiol 96:2984-2994.

McCarson KE and Enna SJ (2014) GABA pharmacology: the search for analgesics. Neurochem Res 39:1948-1963.

Mestre C, Pélissier T, Fialip J, Wilcox G, and Eschalier A (1994) A method to perform direct transcutaneous intrathecal injection in rats. J Pharmacol Toxicol Methods 32:197-200.

Mogil JS (2009) Animal models of pain: progress and challenges. Nat Rev Neurosci 10:283-294.

Montana MC, Conrardy BA, Cavallone LF, Kolber BJ, Rao LK, Greco SC, and Gereau RW, 4th (2011) Metabotropic glutamate receptor 5 antagonism with fenobam: examination of analgesic tolerance and side effect profile in mice. Anesthesiology $\mathbf{1 1 5}$ 1239-1250.

Morrow TJ (2004) Animal models of painful diabetic neuropathy: the STZ rat model. Curr Protoc Neurosci Chapter 9:Unit 9.18.

Obata H, Saito S, Koizuka S, Nishikawa K, and Goto F (2005) The monoaminemediated antiallodynic effects of intrathecally administered milnacipran, a serotonin noradrenaline reuptake inhibitor, in a rat model of neuropathic pain. Anesth Analg 100:1406-1410, table of contents.
Powell DR, DaCosta CM, Gay J, Ding ZM, Smith M, Greer J, Doree D, Jeter-Jones S, Mseeh F, and Rodriguez LA, et al. (2013) Improved glycemic control in mice lacking Sglt1 and Sglt2. Am J Physiol Endocrinol Metab 304:E117-E130.

Santos-Nogueira E, Redondo Castro E, Mancuso R, and Navarro X (2012) RandallSelitto test: a new approach for the detection of neuropathic pain after spinal cord injury. $J$ Neurotrauma 29:898-904.

Shi B, Conner SD, and Liu J (2014) Dysfunction of endocytic kinase AAK1 in ALS. Int J Mol Sci 15:22918-22932.

Snedecor SJ, Sudharshan L, Cappelleri JC, Sadosky A, Desai P, Jalundhwala Y, and Botteman M (2014) Systematic review and meta-analysis of pharmacological therapies for pain associated with postherpetic neuralgia and less common neuropathic conditions. Int J Clin Pract 68:900-918.

Stone LS, German JP, Kitto KF, Fairbanks CA, and Wilcox GL (2014) Morphine and clonidine combination therapy improves therapeutic window in mice: synergy in antinociceptive but not in sedative or cardiovascular effects. PLoS One 9: e109903.

Tang T, Li L, Tang J, Li Y, Lin WY, Martin F, Grant D, Solloway M, Parker L, and Ye $\mathrm{W}$, et al. (2010) A mouse knockout library for secreted and transmembrane proteins. Nat Biotechnol 28:749-755.

Tanimoto-Mori S, Nakazato-Imasato E, Toide K, and Kita Y (2008) Pharmacologic investigation of the mechanism underlying cold allodynia using a new cold plate procedure in rats with chronic constriction injuries. Behav Pharmacol 19:85-90.

Teasell RW, Mehta S, Aubut JA, Foulon B, Wolfe DL, Hsieh JT, Townson AF, and Short C; Spinal Cord Injury Rehabilitation Evidence Research Team (2010) A systematic review of pharmacologic treatments of pain after spinal cord injury. Arch Phys Med Rehabil 91:816-831.

Traub LM (2009) Tickets to ride: selecting cargo for clathrin-regulated internalization. Nat Rev Mol Cell Biol 10:583-596.

Ultanir SK, Hertz NT, Li G, Ge WP, Burlingame AL, Pleasure SJ, Shokat KM, Jan LY, and Jan YN (2012) Chemical genetic identification of NDR1/2 kinase substrates AAK1 and Rabin8 uncovers their roles in dendrite arborization and spine development. Neuron 73:1127-1142.

Wang G, Dai D, Chen X, Yuan L, Zhang A, Lu Y, and Zhang P (2014) Upregulation of neuregulin-1 reverses signs of neuropathic pain in rats. Int $J$ Clin Exp Pathol 7 : $5916-5921$.

Watzman N, Barry H, 3rd, Buckley JP, and Kinnard WJ, Jr (1964) Semiautomatic System for Timing Rotarod Performance. J Pharm Sci 53:1429-1430.

Woolfe G and MacDonald AD (1944) The evaluation of the analgesic action of pethidine hydrochloride (Demerol). J Pharmacol Exp Ther 80:300-307.

Xie K, Qiao F, Sun Y, Wang G, and Hou L (2015) Notch signaling activation is critical to the development of neuropathic pain. BMC Anesthesiol 15:41.

Yaksh TL, Ozaki G, McCumber D, Rathbun M, Svensson C, Malkmus S, and Yaksh MC (2001) An automated flinch detecting system for use in the formalin nociceptive bioassay. J Appl Physiol (1985) 90:2386-2402.

Ye GL, Savelieva KV, Vogel P, Baker KB, Mason S, Lanthorn TH, and Rajan I (2015) Ligation of mouse L4 and L5 spinal nerves produces robust allodynia without major motor function deficit. Behav Brain Res 276:99-110.

Zambrowicz BP and Sands AT (2003) Knockouts model the 100 best-selling drugs: will they model the next 100? Nat Rev Drug Discov 2:38-51.

Zeilhofer HU, Ralvenius WT, and Acuña MA (2015) Restoring the spinal pain gate: GABA(A) receptors as targets for novel analgesics. Adv Pharmacol $\mathbf{7 3}$ $71-96$

Address correspondence to: Dr. Charles F. Albright, Bristol-Myers Squibb, 5 Research Parkway, Wallingford, CT 06492. E-mail: Charlie.Albright@BMS. com or Dr. Brian Zambrowicz, Regeneron Pharmaceuticals, 777 Old Saw Mill River Road, Tarrytown, NY 10591. E-mail: brian.zambrowicz@regeneron.com 